\title{
First-principles studies of beryllium doping of GaN
}

\author{
Chris G. Van de Walle* and Sukit Limpijumnong \\ Xerox Palo Alto Research Center, 3333 Coyote Hill Road, Palo Alto, California 94304
}

Jörg Neugebauer

Fritz-Haber-Institut der Max-Planck-Gesellschaft, Faradayweg 4-6, D-14 195 Berlin-Dahlem, Germany

(Received 12 October 2000; published 8 June 2001)

\begin{abstract}
The structural and electronic properties of beryllium substitutional acceptors and interstitial donors in GaN are investigated using first-principles calculations based on pseudopotentials and density-functional theory. In p-type GaN, Be interstitials, which act as donors, have formation energies comparable to that of substitutional $\mathrm{Be}$ on the Ga site, which is an acceptor. In thermodynamic equilibrium, incorporation of Be interstitials will therefore result in severe compensation. To investigate the kinetics of Be interstitial incorporation and outdiffusion we have explored the total-energy surface. The diffusivity of Be interstitials is highly anisotropic, with a migration barrier in planes perpendicular to the $c$ axis of $1.2 \mathrm{eV}$, while the barrier for motion along the $c$ axis is $2.9 \mathrm{eV}$. We have also studied complex formation between interstitial donors and substitutional acceptors, and between hydrogen and substitutional beryllium. The results for wurtzite $\mathrm{GaN}$ are compared with those for the zinc-blende phase. Consequences for $p$-type doping using Be acceptors are discussed.
\end{abstract}

DOI: 10.1103/PhysRevB.63.245205

PACS number(s): 61.72.-y, 66.30.Jt, 71.55.Eq, 72.80.Ey

\section{INTRODUCTION}

Nitride semiconductors are currently being used in a wide variety of electronic and optoelectronic devices. Controllable doping is essential for all of these devices. For $n$-type doping of nitrides, a number of elements (e.g., $\mathrm{Si}$ ) can be successfully used as dopants, and carrier concentrations exceeding $5 \times 10^{20} \mathrm{~cm}^{-3}$ can routinely be achieved. ${ }^{1}$ The situation is less favorable for $p$-type doping. Magnesium is the acceptor of choice; it can be incorporated in concentrations up to about $10^{20} \mathrm{~cm}^{-3}$, but because of its large ionization energy (210 meV, Ref. 1) the resulting room-temperature hole concentration is only about $10^{18} \mathrm{~cm}^{-3}$, i.e., only about $1 \%$ of $\mathrm{Mg}$ atoms are ionized at room temperature. Increasing the $\mathrm{Mg}$ concentration beyond $10^{20} \mathrm{~cm}^{-3}$ leads to a saturation and decrease in the hole concentration. ${ }^{2}$ In previous work, we have proposed that the $\mathrm{Mg}$ solubility limit is the main cause of this behavior. ${ }^{3}$ The limited conductivity of $p$-type doped layers constitutes an impediment for progress in device applications.

It would be desirable to identify an alternative acceptor that would exhibit higher solubility and/or lower ionization energy. Previous computational studies ${ }^{4-6}$ have addressed a variety of candidate acceptors, including $\mathrm{Li}, \mathrm{Na}, \mathrm{K}, \mathrm{Be}, \mathrm{Zn}$, $\mathrm{Ca}$, and $\mathrm{Cd}$. Only Be emerged as a viable acceptor, exhibiting higher solubility and lower ionization energy than $\mathrm{Mg}$. However, it also emerged that self-compensation may occur due to incorporation of $\mathrm{Be}$ on interstitial sites, where it acts as a donor. ${ }^{5}$ The conclusions of the previous theoretical work seem promising enough, however, to warrant a more comprehensive investigation, which is the subject of the present paper.

Experimentally, Be doping of GaN in MBE (molecular beam epitaxy) has been reported by various groups. ${ }^{7-15}$ These experimental studies have involved both wurtzite and cubic phases of GaN; the cubic phase can be obtained by growth on cubic substrates, under appropriately tailored con- ditions. Another technique for Be incorporation that has been attempted is ion implantation. ${ }^{16,17}$ No electrical conductivity results were reported; characterization was mostly by optical spectroscopy. A photoluminescence (PL) peak just below the band edge of $\mathrm{GaN}$ was found and attributed to Be. Assuming that this PL line results from a Be substitutional acceptor, an acceptor ionization energy can be extracted-but this has resulted in a wide range of values, ranging from 90 to 250 $\mathrm{meV}$, depending on the assumptions made in the analysis. Brandt et al. reported that using oxygen as a codopant along with $\mathrm{Be}$ results in high $p$-type conductivity. ${ }^{18,19}$ Yamamoto and Katayama-Yoshida ${ }^{20}$ proposed an expanation for these codoping results in terms of $\mathrm{Be}_{\mathrm{Ga}}-\mathrm{O}_{\mathrm{N}}-\mathrm{Be}_{\mathrm{Ga}}$ complexes.

The purpose of the present study is to investigate the behavior of $\mathrm{Be}$ in $\mathrm{GaN}$ in detail, with particular focus on the diffusion properties of interstitial Be. Indeed, a thorough understanding of the stability and diffusivity of interstitial Be will allow us to assess the likelihood that interstitial Be will be incorporated during growth - and if it is, whether it might be removed during a subsequent annealing procedure. To address this issue, we have mapped out the total-energy surface for an interstitial Be atom moving through the GaN lattice; this enables us to extract the barriers for Be diffusion. In addition, we have studied the interaction between interstitial $\mathrm{Be}$ donors $\left(\mathrm{Be}_{i n t}\right)$ and substitutional $\mathrm{Be}$ acceptors $\left(\mathrm{Be}_{\mathrm{Ga}}\right)$. Coulomb attraction leads to formation of a complex with a large binding energy $(1.35 \mathrm{eV})$. Because hydrogen is known to play an important role in $p$-type doping of $\mathrm{GaN},{ }^{21-23}$ we have also investigated its interaction with Be acceptors, leading to the formation of $\mathrm{Be}-\mathrm{H}$ complexes with a binding energy of $1.81 \mathrm{eV}$.

We have also performed additional investigations in order to make contact with the above-mentioned experimental studies. Because the experimental studies have involved both wurtzite and cubic phases of $\mathrm{GaN}$, we have performed most of our calculations for both phases. The PL results prompted us to examine the optical ionization energy of the Be accep- 
tor in detail, and we found a large Franck-Condon shift due to an unusual atomic relaxation of the acceptor in its neutral charge state. Because of the interest in codoping with oxygen, finally, we have performed comprehensive calculations for $\mathrm{Be}_{\mathrm{Ga}}-\mathrm{O}_{\mathrm{N}}$ and $\mathrm{Be}_{\mathrm{Ga}}-\mathrm{O}_{\mathrm{N}}-\mathrm{Be}_{\mathrm{Ga}}$ complexes.

The organization of this paper is as follows. In Sec. II, we discuss the computational approach and methods. Section III lists our results for isolated impurities, and Sec. IV contains results for complexes. Section V, finally, contains a discussion and comparison with existing experiments. Section VI summarizes the paper and contains suggestions for future experimental work.

\section{THEORETICAL APPROACH}

\section{A. Pseudopotential density-functional theory}

We use the density-functional theory ${ }^{24}$ in the local density approximation (LDA) and ab initio norm-conserving pseudopotentials, with a plane-wave basis set. ${ }^{25}$ Since the Ga $3 d$ electrons play an important role for the chemical bonding in $\mathrm{GaN}$, they cannot simply be treated as core electrons. Explicitly taking $\mathrm{Ga} 3 d$ as valence electrons is one way to obtain more realistic results, as discussed in detail in Ref. 26. However, the localized nature of the Ga $3 d$ states significantly increases the computational demand, requiring an energy cutoff of at least $60 \mathrm{Ry}$. We have therefore used the so-called "nonlinear core correction,", 27 with an energy cutoff of $40 \mathrm{Ry}$. We have tested this approximation by comparing formation energies for interstitial Be with results obtained using explicit inclusion of $\mathrm{Ga} 3 d$ electrons as valence electrons; the values agree to within $0.1 \mathrm{eV}$.

\section{B. Supercells}

The wurtzite (WZ) structure is the lowest-energy structure of $\mathrm{GaN}$, but the zinc-blende (ZB) structure is only slightly higher in energy $(\approx 10 \mathrm{meV}$ per two-atom unit; Ref. 28$)$ and can be obtained by epitaxial growth on a zinc-blende substrate. We have therefore performed calculations for $\mathrm{Be}$ in both the zinc-blende and the wurtzite structures. As we will see, results for substitutional Be are very similar in both phases, but the properties of interstitial Be are quite different.

All impurity calculations are carried out at the theoretical lattice constant, in order to avoid any spurious relaxations. For wurtzite $\mathrm{GaN}$, we find $a^{\text {th }}=3.089 \AA$ (compared with $a^{\text {expt }}=3.19 \AA$ ). The calculated $c / a$ ratio is 1.633 (experiment 1.627), very close to the ideal $c / a$ ratio of $\sqrt{8 / 3}$. For zinc-blende GaN, we find $a^{\text {th }}=4.370 \AA$, which is (to within $0.001 \AA) \sqrt{2}$ larger than the wurtzite lattice constant.

In order to study the atomic and electronic structure of an impurity in the GaN crystal, we construct an artificial unit cell (supercell) composed of several primitive GaN unit cells and containing one impurity. The larger the supercell size, the closer our results will be to the case of a single, isolated impurity, because interactions between impurities in neighboring supercells are suppressed. The computation time, however, increases as $N^{2} \ln N$ where $N$ is the number of atoms in the supercell. It is therefore imperative to choose the smallest possible cell size that produces reliable results

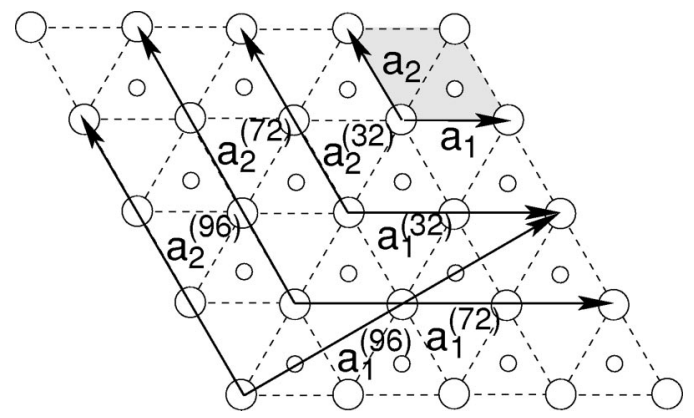

FIG. 1. Top view (along [0001] direction) of the GaN wurtzite structure: small circles represent nitrogen, large circles gallium. The shaded area corresponds to the primitive unit cell. The translation vectors for the primitive unit cell and for the 32-, 72-, and 96-atom supercells are also shown.

for the problem under investigation. We have performed calculations for several different supercell sizes, in the process checking convergence.

For the wurtzite structure, we have employed 32-, 72-, and 96-atom supercells. The 32-atom supercell is composed of eight wurtzite GaN primitive unit cells (each containing four atoms), such that each translation vector of the supercell is doubled from that of the basic unit cell. If we choose the lattice translation vectors for wurtzite $\mathrm{GaN}$ to be $\mathbf{a}_{1}$ $=(a, 0,0), \mathbf{a}_{2}=(-a / 2, \sqrt{3} a / 2,0)$, and $\mathbf{a}_{3}=(0,0, c)$ (see Fig. $1)$, the translation vectors for the 32-atom supercell are defined as $\mathbf{a}_{i}^{(32)}=2 \mathbf{a}_{i}$, where $i=1,2,3$. For the 72-atom supercell, the translation vectors are $\mathbf{a}_{1}^{(72)}=3 \mathbf{a}_{1}, \mathbf{a}_{2}^{(72)}=3 \mathbf{a}_{2}$, and $\mathbf{a}_{3}^{(72)}=2 \mathbf{a}_{1}$. As can be noted from Fig. 1, both 32- and 72atom supercells suffer from the problem that the separation between impurities in neighboring supercells (imagined to be located at the center of the supercell) is quite different when measured along different directions. We have therefore considered a 96-atom supercell that has translation vectors that are mutually perpendicular, leading to a cell with orthorhombic symmetry. The translation vectors of this 96-atom supercell are $\mathbf{a}_{1}^{(96)}=4 \mathbf{a}_{1}+2 \mathbf{a}_{2}, \mathbf{a}_{2}^{(96)}=3 \mathbf{a}_{2}$, and $\mathbf{a}_{3}^{(96)}=2 \mathbf{a}_{3}$.

For the zinc-blende structure, we have used 32- and 64atom supercells, which have been extensively discussed in the context of point-defect calculations in zinc-blende semiconductors. The 32-atom supercell has bcc symmetry, while the 64-atom cell is cubic, consisting of the conventional eight-atom cubic unit cell of the zinc-blende structure doubled in each direction.

Within the supercell, we allow relaxation of several shells of host atoms around the impurity. For wurtzite, 24 host atoms were relaxed in the 32-atom cells. In the 96-atom cell, at least 46 host atoms (plus the impurity) were allowed to relax, including all atoms within $4.8 \AA$ of any impurity. For a substitutional impurity, this corresponds to seven shells of atoms. In the zinc-blende 64-atom cell, the same relaxation radius leads to at least 44 atoms being relaxed (five shells).

Results of our convergence tests will be discussed in Sec. III A. The tests indicate that, for zinc blende, 32-atom and 64-atom supercells yield very similar results, indicating convergence. For wurtzite, the absolute values of formation energies are not yet converged in a 32-atom cell. The 96-atom 
cell results are expected to be converged (for substitional impurities, these results are very close to those for the $\mathrm{ZB}$ 64-atom cells). However, 32-atom wurtzite cell calculations are very useful to obtain energy differences between different positions of the impurity in the lattice; we found these energy differences to be within $0.3 \mathrm{eV}$ of those calculated in the 96-atom cell. Since mapping out the total-energy surface describing the motion of interstitial $\mathrm{Be}$ through the lattice requires a large number of calculations, we have used the 32-atom cell for that purpose.

\section{Special k points}

Brillouin-zone integrations were carried out following the Monkhorst-Pack scheme ${ }^{29}$ with a regularly spaced mesh of $n \times n \times n$ points in the reciprocal unit cell shifted from the origin (to avoid picking up the $\Gamma$ point as one of the sampling points). Symmetry reduces this set to a set of points in the irreducible part of the Brillouin zone. Convergence tests indicate that for zinc blende the $2 \times 2 \times 2$ sampling yields total energies that are converged to better than $0.1 \mathrm{eV}$ in both 32-atom and 64-atom supercells. For the 32-atom wurtzite supercell with $\mathrm{Be}_{i n t}^{2+}$, a $2 \times 2 \times 2$ set does not yield fully converged results; energy differences are obtained reliably, however. We also compared the total energies calculated using $1 \times 1 \times 1$ and $2 \times 2 \times 2$ sets. The former leads to only one irreducible $\mathbf{k}$ point while the latter leads to three irreducible $\mathbf{k}$ points. Although the absolute value of the total energy differs significantly between the two calculations, the energy differences between different $\mathrm{Be}_{\text {int }}$ locations change by less than $0.1 \mathrm{eV}$. The calculations required to explore the totalenergy surface were therefore carried out using only one special $\mathbf{k}$ point. In the 96 -atom wurtzite cell, finally, the $2 \times 2$ $\times 2$ k-point mesh produces fully converged results.

\section{Charge states}

Beryllium substituting on the $\mathrm{Ga}$ site in $\mathrm{GaN}$ is a single acceptor; we therefore study two charge states, $\mathrm{Be}_{\mathrm{Ga}}^{-}$and $\mathrm{Be}_{\mathrm{Ga}}^{0}$. In the case of a shallow acceptor such as $\mathrm{Be}_{\mathrm{Ga}}$ the level introduced in the Kohn-Sham band structure due to the presence of the impurity is merely a perturbation of the host band structure. This "acceptor level" therefore exhibits essentially the same dispersion as the uppermost valence band. In the negative charge state, the acceptor level is filled-but in the neutral charge state, one electron is removed from this level. For a true, isolated acceptor (corresponding to a calculation in a very large supercell), the electron would be removed from the top of the valence band, at the $\Gamma$ point. But in our finite-size supercells the electron is actually taken out of the highest occupied Kohn-Sham level at the special $\mathbf{k}$ points, where the band energy is lower than at the $\Gamma$ point. A correction is therefore needed, obtained from the energy differences between the highest occupied state at the $\Gamma$ point and the special $\mathbf{k}$ points. The magnitude of this correction ranges from $\sim 0.2 \mathrm{eV}$ in the 96-atom cell to $\sim 0.5 \mathrm{eV}$ in the 32-atom cells. This correction term, which we call $E_{\text {corr }}$ and which has been defined as a positive number, has been taken into account in all our results for neutral acceptors. ${ }^{30}$
As we will see in the next section, the formation energy of non-neutral impurities takes into account that electrons are exchanged with the Fermi level. The Fermi level $E_{F}$ is referenced to the valence-band maximum in the bulk, i.e., $E_{F}$ $=0$ at the top of the valence band $\left(E_{v}\right)$ in bulk GaN. We obtain $E_{v}$ by calculating the position of the valence-band maximum in $\mathrm{GaN}$ with respect to the average electrostatic potential. We need to bear in mind, however, that the average electrostatic potential in our defect supercells is not the same as that in bulk GaN; indeed, our first-principles calculations for periodic structures (which fill all space) do not provide an absolute reference for the electrostatic potential, due to the long-range nature of the Coulomb potential. An explicit alignment procedure is thus needed between the electrostatic potential in the defect supercell and the electrostatic potential in the bulk. This problem is similar to that of calculating heterojunction band offsets, and similar techniques can be used to address these issues. ${ }^{31}$ Here we have chosen to align the electrostatic potentials by inspecting the potential in the supercell far from the impurity and aligning it with the electrostatic potential in bulk GaN. This leads to a shift in the reference level, $\Delta V$, which needs to be added to $E_{v}$ in order to obtain the correct alignment. The resulting shifts are taken into account in our expressions for formation energies in the next section.

Interstitial $\mathrm{Be}$ atoms in $\mathrm{GaN}$ are double donors. In this work we have focused on the +2 charge state $\left(\mathrm{Be}_{i n t}^{2+}\right)$, but we make some comments about other charge states in Sec. III C 2.

Finally, we have also studied the $\left(\mathrm{Be}_{\mathrm{Ga}}-\mathrm{Be}_{i n t}\right)$ complex; from the charge states of the interstitial and substitutional species, it can be inferred that this complex has a charge state of +1 . The neutral charge state is discussed in Sec. IV A.

\section{E. Formation energies}

The formation energy $E^{f}$ determines the concentration $c$ of the impurity in the semiconductor, through the expression

$$
c=N_{\text {sites }} \exp \left(-E^{f} / k T\right),
$$

where $N_{\text {sites }}$ is the number of sites in the lattice (per unit volume) where the impurity can be incorporated, $k$ is Boltzmann's constant, and $T$ is the temperature. Equation (1) in principle holds only in thermodynamic equilibrium. Growth of a semiconductor is obviously a nonequilibrium process; however, many growth environments involve high enough temperatures to ensure reasonable mobility of impurities and defects at or near the surface, establishing conditions that approximate equilibrium. Furthermore, even in nonequilibrium conditions the formation energy of an impurity (or defect) is a useful concept, since configurations with high formation energies will obviously be difficult to incorporate (or will attempt to evolve to a lower-energy state).

The formation energies of $\mathrm{Be}_{i n t}^{2+}, \mathrm{Be}_{\mathrm{Ga}}^{0}, \mathrm{Be}_{\mathrm{Ga}}^{-}$, and $\left(\mathrm{Be}_{\mathrm{Ga}}-\mathrm{Be}_{i n t}\right)^{+}$are defined as 


$$
\begin{aligned}
& E^{f}\left[\mathrm{Be}_{i n t}^{2+}\right]=E_{t o t}\left[\mathrm{Be}_{i n t}^{2+}\right]-E_{\text {tot }}[\mathrm{GaN}, \text { bulk }]-\mu_{\mathrm{Be}} \\
& +2\left[E_{F}+E_{v}+\Delta V\left(\mathrm{Be}_{i n t}\right)\right], \\
& E^{f}\left[\mathrm{Be}_{\mathrm{Ga}}^{0}\right]=E_{\text {tot }}\left[\mathrm{Be}_{\mathrm{Ga}}^{0}\right]-E_{\text {tot }}[\mathrm{GaN}, \text { bulk }]-\mu_{\mathrm{Be}}+\mu_{\mathrm{Ga}} \\
& -E_{\text {corr }} \text {, } \\
& E^{f}\left[\mathrm{Be}_{\mathrm{Ga}}^{-}\right]=E_{\text {tot }}\left[\mathrm{Be}-\mathrm{Ga}_{\text {Gat }}^{-}\right]-E_{t o n}[\mathrm{GaN}, \text { bulk }]-\mu_{\mathrm{Be}}+\mu_{\mathrm{Ga}} \\
& -\left[E_{F}+E_{v}+\Delta V\left(\mathrm{Be}_{\mathrm{Ga}}\right)\right], \\
& E^{f}\left[\left(\mathrm{Be}_{\mathrm{Ga}}-\mathrm{Be}_{i n t}\right)^{+}\right]=E_{t o t}\left[\left(\mathrm{Be}_{\mathrm{Ga}}-\mathrm{Be}_{i n t}\right)^{+}\right] \\
& -E_{\text {tot }}[\mathrm{GaN}, \text { bulk }]-2 \mu_{\mathrm{Be}}+\mu_{\mathrm{Ga}} \\
& +\left[E_{F}+E_{v}+\Delta V\left(\mathrm{Be}_{\mathrm{Ga}}-\mathrm{Be}_{i n t}\right)\right] .
\end{aligned}
$$

$E_{\text {tot }}[X]$ is the total energy derived from a supercell calculation with one impurity $X$ in the cell, and $E_{\text {tot }}[\mathrm{GaN}$, bulk] is the total energy for the equivalent supercell containing only bulk GaN. $\mu_{\mathrm{Ga}}$ and $\mu_{\mathrm{Be}}$ are the chemical potentials of $\mathrm{Ga}$ and Be, respectively. $E_{F}$ is the Fermi level, referenced to the valence-band maximum in the bulk. Due to the choice of this reference, we need to explicitly include the energy of the bulk valence-band maximum, $E_{v}$, in our expressions for formation energies of charged states. As discussed in Sec. II D, we also need to add a correction term $\Delta V$ to align the reference potential in our defect supercell with that in the bulk. Finally, the correction term $E_{\text {corr }}$ that appears in the formation energy of $\mathrm{Be}_{\mathrm{Ga}}^{0}$ was discussed in Sec. II D.

The chemical potentials depend on the experimental growth conditions, which can be either $\mathrm{Ga}$ rich or $\mathrm{N}$ rich. For the Ga-rich case, we use $\mu_{\mathrm{Ga}}=\mu_{\mathrm{Ga} \text { [bulk] }}$ to place an upper limit on $\mu_{\mathrm{Ga}}$; this places a lower limit on $\mu_{\mathrm{N}}$, calculated from

$$
\mu_{\mathrm{N}}^{\min }=E_{\text {tot }}[\mathrm{GaN}]-\mu_{\mathrm{Ga}},
$$

where $E_{\text {tot }}[\mathrm{GaN}]$ is the total energy of a two-atom unit of bulk GaN, calculated for the structurally optimized wurtzite structure. For the N-rich case, the upper limit on $\mu_{\mathrm{N}}$ is given by $\mu_{\mathrm{N}}=\mu_{\left.\mathrm{N}_{[} \mathrm{N}_{2}\right]}$, i.e., the energy of $\mathrm{N}$ in a $\mathrm{N}_{2}$ molecule at $T=0$; this yields a lower limit on $\mu_{\mathrm{Ga}}$ calculated from

$$
\mu_{\mathrm{Ga}}^{\min }=E_{t o t}[\mathrm{GaN}]-\mu_{\mathrm{N}} .
$$

The total energy of GaN can also be expressed as

$$
E_{\text {tot }}[\mathrm{GaN}]=\mu_{\mathrm{Ga}[\mathrm{bulk}]}+\mu_{{\mathrm{N}\left[\mathrm{N}_{2}\right]}}+\Delta H_{f}[\mathrm{GaN}],
$$

where $\Delta H_{f}[\mathrm{GaN}]$ is the enthalpy of formation, which is negative for a stable compound. Our calculated value for $\Delta H_{f}[\mathrm{GaN}]$ is $-0.48 \mathrm{eV}$ (expt. $-1.17 \mathrm{eV}$; Ref. 32). By combining Eq. (6) or Eq. (7) with Eq. (8), we observe that the host chemical potentials vary over a range corresponding to the magnitude of the enthalpy of formation of GaN.

For $\mathrm{Be}$, the upper bound on the chemical potential arises from the solubility-limiting phase, which is $\mathrm{Be}_{3} \mathrm{~N}_{2}$. There is no lower bound on $\mu_{\mathrm{Be}}$, minus infinity corresponding to the total absence of $\mathrm{Be}$ from the growth environment. Equilibrium with $\mathrm{Be}_{3} \mathrm{~N}_{2}$ implies

$$
3 \mu_{\mathrm{Be}}+2 \mu_{\mathrm{N}}=3 \mu_{\mathrm{Be}[\text { bulk }]}+2 \mu_{\left.\mathrm{N}_{[} \mathrm{N}_{2}\right]}+\Delta H_{f}\left[\mathrm{Be}_{3} \mathrm{~N}_{2}\right],
$$

where $\Delta H_{f}\left[\mathrm{Be}_{3} \mathrm{~N}_{2}\right]$ is the calculated enthalpy of formation of $\mathrm{Be}_{3} \mathrm{~N}_{2}$, which is $-5.5 \mathrm{eV} .^{33}$ The experimental value of $\Delta H_{f}\left[\mathrm{Be}_{3} \mathrm{~N}_{2}\right]$ is $-6.11 \mathrm{eV} \cdot{ }^{34}$ Equation (9) allows us to relate $\mu_{\mathrm{Be}}$ to $\mu_{\mathrm{N}}$, assuming equilibrium with $\mathrm{Be}_{3} \mathrm{~N}_{2}$. Combining this information with the expression for the formation energy of $\mathrm{Be}_{\mathrm{Ga}}$ [Eq. (3)], we find that the lowest formation energy (and hence the highest concentration of $\mathrm{Be}_{\mathrm{Ga}}$, i.e., the solubility limit) will occur under nitrogen-rich conditions, i.e., when $\mu_{\mathrm{N}}=\mu_{\mathrm{N}\left[\mathrm{N}_{2}\right]} \cdot{ }^{35}$

Ultimately, one wants to calculate concentrations of native defects or impurities. For that purpose, one would choose a particular set of atomic chemical potentials (corresponding to the growth conditions), and substitute the formation energies from Eqs. (2)-(5) in the expression for concentrations, Eq. (1). This still leaves the Fermi level as a free parameter. This remaining variable is fixed by imposing the condition of charge neutrality: the total charge resulting from charged defects or impurities, along with the charge due to any free carriers in valence and conduction bands, needs to be equal to zero. This leads to a unique position for the Fermi level.

It turns out to be very informative to explicitly plot the dependence of formation energies on Fermi levels: this immediately provides insight into the electrical activity (donor or acceptor character), and shows whether certain defects will act as compensating centers. We will therefore present our results in this format.

\section{F. Thermal and optical ionization energies}

The thermal ionization energy $E_{A}$ of the $\mathrm{Be}_{\mathrm{Ga}}$ substitutional acceptor is defined as the transition energy between the neutral and negative charge states of the dopant [sometimes the notation $\epsilon(0 /-)$ is also used for this quantity]. This transition energy is defined as the Fermi-level position where the formation energies of these two charge states are equal, i.e.,

$$
E^{f}\left[\mathrm{Be}_{\mathrm{Ga}}^{-}\right]\left(E_{F}=E_{A}\right)=E^{f}\left[\mathrm{Be}_{\mathrm{Ga}}^{0}\right] .
$$

From Eqs. (3) and (4), it then follows that

$$
\begin{aligned}
E_{A}= & E^{f}\left[\mathrm{Be}_{\mathrm{Ga}}^{-}\right]\left(E_{F}=0\right)-E^{f}\left[\mathrm{Be}_{\mathrm{Ga}}^{0}\right] \\
= & E_{\text {tot }}\left[\mathrm{Be} e_{\mathrm{Ga}}^{-}\right]-E_{\text {tot }}\left[\mathrm{Be}_{\mathrm{Ga}}^{0}\right] \\
& +E_{\text {corr }}-E_{v}-\Delta V\left(\mathrm{Be}_{\mathrm{Ga}}\right) .
\end{aligned}
$$

For purposes of defining the thermal ionization energy, it is implied that for each charge state the atomic structure is relaxed to its equilibrium configuration. The atomic positions in these equilibrium configurations are not necessarily the same for both charge states. Indeed, we will see that they are quite different for the neutral and negative charge states of $\mathrm{Be}_{\mathrm{Ga}}$.

This difference leads to an interesting and sizable effect when the ionization energy of the dopant is determined optically. Assume the following simplified picture of a photoluminescence experiment. The exciting light creates electron-hole pairs. The holes can be trapped at $\mathrm{Be}_{\mathrm{Ga}}^{-}$centers, 


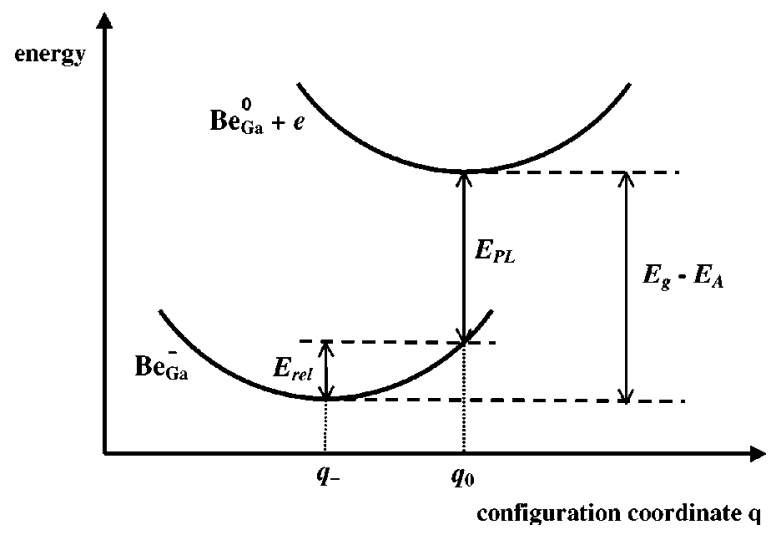

FIG. 2. Schematic configuration coordinate diagram illustrating the difference between thermal and optical ionization energies. The curve for $\mathrm{Be}_{\mathrm{Ga}}^{0}$ is vertically displaced from that for $\mathrm{Be}_{\mathrm{Ga}}^{-}$assuming the presence of an electron in the conduction band. $E_{\text {rel }}$ is the Franck-Condon shift, i.e., the relaxation energy that can be gained, in the negative charge state, by relaxing from configuration $q_{0}$ (equilibrium configuration for the neutral charge state) to configuration $q_{-}$(equilibrium configuration for the negative charge state).

turning them into $\mathrm{Be}_{\mathrm{Ga}}^{0}$. Using our definition of the thermal ionization energy $E_{A}$, the equilibrium configuration of the $\mathrm{Be}_{\mathrm{Ga}}^{0}+e$ state (where $e$ is an electron at the bottom of the conduction band) is $E_{g}-E_{A}$ higher than the equilibrium configuration of $\mathrm{Be}_{\mathrm{Ga}}^{-}$, where $E_{g}$ is the band gap. Electrons in the conduction band can then recombine with the hole on the acceptor, as illustrated in Fig. 2. This leads to emission of a photon with energy $E_{P L}$. During this emission process, the atomic configuration of the acceptor remains fixed-i.e., in the final state, the acceptor is in the negative charge state, but with a structure (configuration coordinate $q_{0}$ ) that is the same as that for the neutral charge state. The difference between the energy of this configuration and that of the equilibrium configuration $q_{-}$is the relaxation energy $E_{r e l}$ (the Franck-Condon shift). From Fig. 2 it is clear that $E_{P L}=E_{g}$ $-E_{A}-E_{r e l}$. If the optical ionization energy $E_{A}^{o p t}$ is defined as the energy difference between the band gap and the PL line, we find that $E_{A}^{o p t}=E_{g}-E_{P L}=E_{A}+E_{r e l}$. This simplified picture ignores excitonic effects, etc., but it does show that the ionization energy extracted from an optical measurement should be larger than the thermal ionization energy $E_{A}$ by an amount $E_{r e l}$. If the atomic configuration in the two charge states is significantly different (as will turn out to be the case for $\left.\mathrm{Be}_{\mathrm{Ga}}\right), E_{\text {rel }}$ can be sizable.

\section{RESULTS FOR ISOLATED IMPURITIES}

\section{A. The substitutional $\mathrm{Be}_{\mathrm{Ga}}$ acceptor in zinc-blende and wurtzite GaN}

The formation energies as a function of $E_{F}$ are shown in Fig. 3 for Ga-rich and N-rich conditions. As discussed in Sec. II E, N-rich conditions favor the incorporation of $\mathrm{Be}$ on Ga sites, leading to the largest possible $\mathrm{Be}_{\mathrm{Ga}}$ concentration (the solubility limit). Moving toward Ga-rich conditions does not alter our qualitative conclusions; numerical results for any choice of the chemical potentials can always be ob-
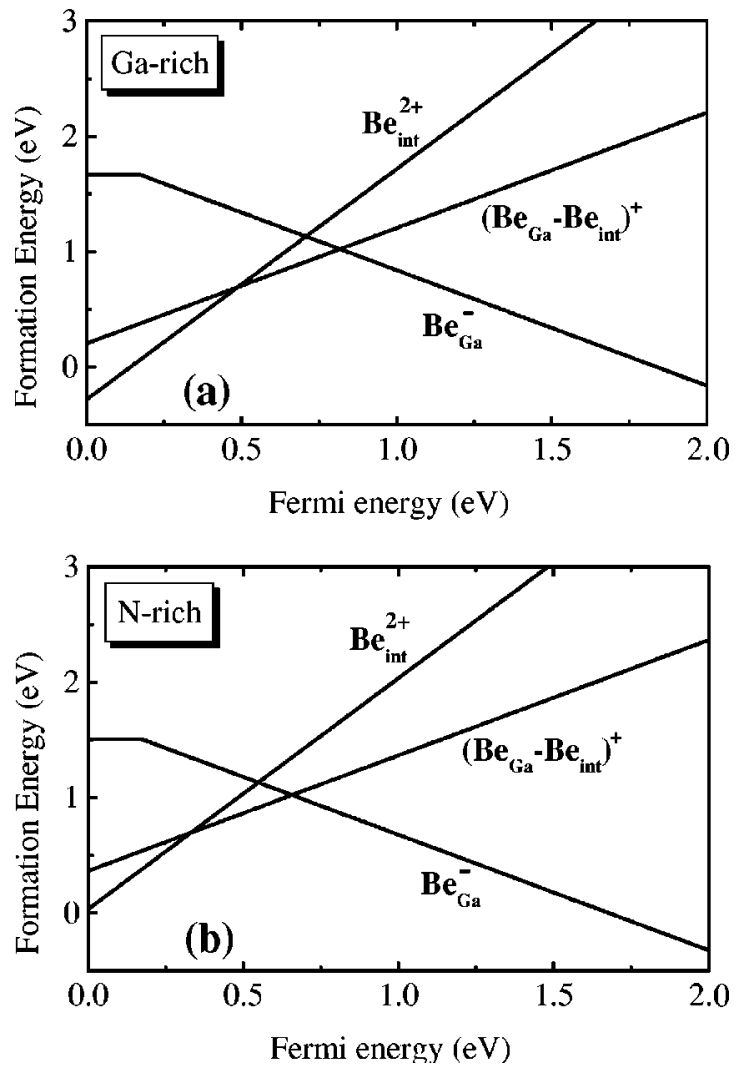

FIG. 3. Calculated defect formation energies as a function of Fermi level for various configurations of Be impurities in wurtzite $\mathrm{GaN}$, under (a) Ga-rich conditions and (b) N-rich conditions.

tained, of course, by referring to the expressions for formation energies in Eqs. (2)-(5). In particular, by combining these expressions with the information on chemical potentials in Eqs. (8) and (9), we find that by moving from N-rich to Ga-rich conditions the formation energy of $\mathrm{Be}_{\mathrm{Ga}}$ increases by $\frac{1}{3}\left|\Delta H_{f}[\mathrm{GaN}]\right|$, while the formation energy of $\mathrm{Be}_{i n t}$ decreases by $\frac{2}{3}\left|\Delta H_{f}[\mathrm{GaN}]\right|$. Consequences of these results for optimizing growth conditions will be discussed in Sec. V.

The formation energies are also summarized in Table I; the numbers given there assume the Fermi level is located at the top of the valence band. We notice that, for the substitu-

TABLE I. Calculated formation energies for Be substitutional acceptors $\left(\mathrm{Be}_{\mathrm{Ga}}\right)$, Be interstitial donors $\left(\mathrm{Be}_{i n t}\right)$, and $\mathrm{Be}_{\mathrm{Ga}}-\mathrm{Be}_{i n t}$ complexes in wurtzite (WZ) and zinc-blende (ZB) GaN. The formation energies [see Eqs. (2)-(5)] are given for $E_{F}=0$, i.e., $E_{F}$ located at the top of the valence band, and for nitrogen-rich conditions. The dependence of formation energies on Fermi level is shown in Fig. 3.

\begin{tabular}{llc}
\hline \hline & \multicolumn{2}{c}{ Formation energies (eV) } \\
& $\mathrm{WZ}$ & $\mathrm{ZB}$ \\
\hline $\mathrm{Be}_{\mathrm{Ga}}^{-}$ & 1.68 & 1.67 \\
$\mathrm{Be}_{\mathrm{Ga}}^{0}$ & 1.51 & 1.47 \\
$\mathrm{Be}_{i n t}^{2+}$ & 0.04 & -0.57 \\
$\left(\mathrm{Be}_{\mathrm{Ga}}-\mathrm{Be}_{i n t}\right)^{+}$ & 0.37 & -0.01 \\
\hline \hline
\end{tabular}


TABLE II. Relaxations around Be substitutional acceptors $\left(\mathrm{Be}_{\mathrm{Ga}}\right)$ in wurtzite $(\mathrm{WZ})$ and zincblende (ZB) GaN. $\Delta d_{\mathrm{Be}}$ denotes the displacement of the $\mathrm{Be}$ atom from its nominal lattice site, expressed as a percentage of the bulk Ga-N bond length. $\Delta d_{\|}$and $\Delta d_{\perp}$ denote the percentage of change in the Be-N bond length, again expressed referenced to the bulk Ga-N bond length. For wurtzite, the symbol $\|$ denotes the direction parallel to the $c$ axis ([0001]); the other bond directions are denoted by the symbol $\perp$. For zinc blende, the symmetry around the impurity is lowered to $C_{3 v}$, with $\|$ denoting the particular [111] direction in which the impurity is displaced, and $\perp$ denoting the other $\langle 111\rangle$ directions.

\begin{tabular}{lcccc}
\hline \hline & \multicolumn{2}{c}{$\mathrm{Be}_{\mathrm{Ga}}^{-}$} & \multicolumn{2}{c}{$\mathrm{Be}_{\mathrm{Ga}}^{0}$} \\
& $\mathrm{WZ}$ & $\mathrm{ZB}$ & $\mathrm{WZ}$ & $\mathrm{ZB}$ \\
\hline$\Delta d_{\mathrm{Be}}$ & $0.3 \%$ & $0 \%$ & $10.7 \%$ & $11.4 \%$ \\
$\Delta d_{\|}$ & $-4.6 \%$ & $-4.9 \%$ & $12.2 \%$ & $14.5 \%$ \\
$\Delta d_{\perp}$ & $-5.4 \%$ & $-4.9 \%$ & $-8.7 \%$ & $-9.3 \%$ \\
\hline \hline
\end{tabular}

tional acceptor $\mathrm{Be}_{\mathrm{Ga}}$, the results for wurtzite and zinc-blende structures are remarkably similar, the differences being smaller than the calculational error bars. The ionization energies, as defined in Eq. (11), are $0.17 \mathrm{eV}$ for the wurtzite structure and $0.20 \mathrm{eV}$ for zinc blende.

Table II summarizes our results for the atomic configuration of substitutional Be in wurtzite and zinc-blende GaN. The atomic configurations of $\mathrm{Be}_{\mathrm{Ga}}^{-}$and $\mathrm{Be}_{\mathrm{Ga}}^{0}$ in wurtzite $\mathrm{GaN}$ are also schematically illustrated in Fig. 4. This figure, as well as all subsequent schematic representations of atomic structures, is based on calculated atomic coordinates obtained from a 96-atom supercell calculation. The substitutional $\mathrm{Be}$ atom is surrounded by four $\mathrm{N}$ neighbors. A contraction of the Be-N bond length is expected since the atomic radius of $\mathrm{Be}$ is smaller than that of $\mathrm{Ga}$ : the covalent radius of Be is $0.90 \AA$, while that of $\mathrm{Ga}$ is $1.26 \AA{ }^{36}$ Based on this difference, and using a bond length of $1.95 \AA$ for bulk GaN,
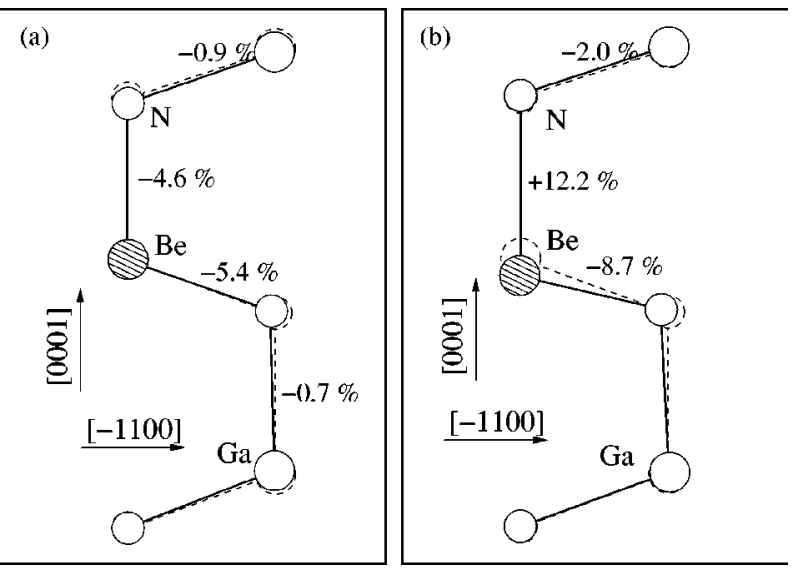

FIG. 4. Schematic representation of atomic positions in the (11520) plane for (a) $\mathrm{Be}_{\mathrm{Ga}}^{-}$and (b) $\mathrm{Be}_{\mathrm{Ga}}^{0}$ in wurtzite GaN. Large circles represent $\mathrm{Ga}$ atoms, medium circles $\mathrm{N}$ atoms, and the hatched circle represents Be. Dashed circles indicate ideal atomic positions, dashed lines bonds in the ideal lattice. The numbers denote the percentage of change in the bond lengths, referenced to the bulk Ga-N bond length (as in Table II). we would expect a contraction of the Be-N bond length by $18 \%$ compared to the Ga-N bond length. This is indeed observed in the compound $\mathrm{Be}_{3} \mathrm{~N}_{2}$, where the bond lengths are 1.50-1.64 $\AA, 16-23 \%$ shorter than in GaN. Using ionic $\operatorname{radii}^{37}\left(0.27 \AA\right.$ for $\mathrm{Be}^{2+}$ and $0.47 \AA$ for $\left.\mathrm{Ga}^{3+}\right)$, we would expect a contraction of about $10 \%$. The actual magnitude of the contraction of the Be-N bond length as calculated for a $\mathrm{Be}_{\mathrm{Ga}}^{-}$impurity in $\mathrm{GaN}$ is less than $6 \%$. The Be-N bond length is thus somewhat longer than is optimal for Be-N, reflecting the fact that the energy cost associated with moving the surrounding $\mathrm{N}$ atoms (as well as further shells of atoms) is quite large. As discussed in Ref. 5, this makes substitutional Be in GaN less energetically favorably than could be hoped based on the large Be-N bond strength. In spite of this unfavorable size match, it was observed that the formation energy of $\mathrm{Be}_{\mathrm{Ga}}$ compares favorably with that of other substitutional acceptors. These observations about size mismatch do show, however, that strategies aimed at relieving the lattice strain associated with incorporating substitutional Be could have an important impact. ${ }^{38}$

In the negative charge state, the $\mathrm{Be}$ atom is located essentially on the substitutional lattice site. In the neutral charge state the situation is very different. Here the Be atom undergoes a sizable relaxation off the nominal lattice site, by more than $10 \%$ of the bond length. We also note that the $d_{\|}$bond length becomes very large, indicating that the bond with one of the $\mathrm{N}$ neighbors is significantly weakened. Simultaneously, the bonds with the other three $\mathrm{N}$ neighbors contract by almost $10 \%$.

The large off-center displacement of $\mathrm{Be}_{\mathrm{Ga}}^{0}$ was found in calculations for supercells of different sizes and shapes, and occurs in both the wurtzite and the zinc-blende structures (see Table II). In the wurtzite structure, the configuration listed in Table II and depicted in Fig. 4 involves the Be atom moving along the $c$ axis. The configuration in which $\mathrm{Be}_{\mathrm{Ga}}^{0}$ moves along the direction of one of the other Be-N bonds is very close in energy (within $0.03 \mathrm{eV}$ ), with very similar relaxations around the Be atom. This tendency of Be to form stronger bonds with three of its nitrogen neighbors is not unexpected. Consider, for instance, the atomic structure of the hexagonal modification of $\mathrm{Be}_{3} \mathrm{~N}_{2}$ [Ref. 39]: some of the $\mathrm{Be}$ atoms are surrounded by a deformed tetrahedron of $\mathrm{N}$ atoms, while others are surrounded by a triangle of nitrogen atoms. It thus seems to be energetically quite acceptable for $\mathrm{Be}$ to form strong bonds with only three of its four $\mathrm{N}$ neighbors. This tendency will also emerge when we inspect bonding configurations for interstitial Be.

Since the equilibrium configuration for the neutral acceptor is so different from that for the negative charge state, we expect the relaxation energy $E_{r e l}$ (as defined in Sec. II F and Fig. 2) to be quite sizable. $E_{r e l}$ is the energy difference, for $\mathrm{Be}_{\mathrm{Ga}}^{-}$, between the atomic configuration that yields the minimum energy for the neutral charge state (i.e., with large lattice relaxation) and the atomic configuration that yields the minimum energy for the negative charge state (i.e., with $\mathrm{Be}$ on the ideal lattice site); this yields $E_{r e l}=0.10 \mathrm{eV}$. Following the discussion in Sec. II F, we thus expect the ionization energy of the acceptor as determined optically to be signifi- 
cantly higher than the thermal ionization energy. Consequences of this result for experimental observations will be discussed in Sec. V B.

The large off-center relaxation of the Be acceptor (at least in the neutral charge state) is reminiscent of the large lattice relaxations experienced by donor atoms in the so-called $D X$ centers; ${ }^{40}$ in this process, the shallow donor $\left(d^{+}\right)$is converted into a deep acceptor $\left(D X^{-}\right)$. A similar mechanism for acceptors would involve placing the acceptor in a positive charge state $\left(A X^{+}\right)$and letting it undergo a large lattice relaxation. We have carefully examined whether such a mechanism can occur for $\mathrm{Be}$, but have found no evidence for stability of a $\mathrm{Be}_{\mathrm{Ga}}^{+}$configuration.

We have examined the stability of $\mathrm{Be}_{\mathrm{Ga}}$ by displacing the $\mathrm{Be}$ atom from the substitutional site; such a displacement would be the first step in a process where the Be would move away from the substitutional site, forming a Be interstitial and leaving a $\mathrm{Ga}$ vacancy behind. We investigated various configurations in which a Be interstitial was placed in the vicinity of a $\mathrm{Ga}$ vacancy. When the overall system was placed in a negative charge state (corresponding to the $\mathrm{Be}_{\mathrm{Ga}}^{-}$ reference state), the system immediately relaxed back, without any barrier, to the $\mathrm{Be}_{\mathrm{Ga}}$ configuration. For other charge states, we found it impossible to obtain converged calculations for the system with Be moved off the substitutional site, indicating the instability of such configurations. Although it is relatively easy to form Be interstitials (see Sec. III C), the energy cost to create a Ga vacancy is prohibitively high, particularly in $p$-type $\mathrm{GaN} ;{ }^{41}$ it is therefore exceedingly unlikely that substitutional Be would leave the Ga site.

\section{B. Interstitial $\mathrm{Be}\left(\mathrm{Be}_{i n t}\right)$ in zinc-blende $\mathrm{GaN}$}

The formation energy of the interstitial species $\mathrm{Be}_{i n t}^{2+}$ in zinc-blende GaN is listed in Table I. This formation energy is negative. One should keep in mind, however, that the formation energy depends on the Fermi-level position [see Eq. (2)]; the value given in Table I is for $E_{F}=0$, i.e., $E_{F}$ positioned at the valence-band maximum. The formation energy increases (and becomes positive) when the Fermi level is higher in the band gap (see Fig. 3). As described in Sec. II E, the final Fermi-level position is determined by charge neutrality, and this position will always correspond to positive formation energies. Table I shows that the formation energy is higher in the wurtzite structure, though still low enough to be a concern for compensation, as discussed in Sec. III C and Sec. V.

The global minimum for $\mathrm{Be}_{i n t}^{2+}$ in $\mathrm{ZB} \mathrm{GaN}$ is at the tetrahedral interstitial site surrounded by nitrogen atoms $\left(T_{d}^{\mathrm{N}}\right)$. The Be atom is essentially at the center of the cage, and the relaxation of the $\mathrm{N}$ atoms is such that the $\mathrm{Be}-\mathrm{N}$ distance is approximately $1.73 \AA$; the $\mathrm{N}$ atoms thus move toward Be by more than $0.20 \AA$. Interstitial diffusion in the zinc-blende structure proceeds via a path through hexagonal rings, with an intermediate position at the tetrahedral interstitial site surrounded by $\mathrm{Ga}$ atoms $\left(T_{d}^{\mathrm{Ga}}\right)$. The energy difference between $T_{d}^{\mathrm{Ga}}$ and $T_{d}^{\mathrm{N}}$ can thus serve as an estimate for the diffusion activation energy; this value is equal to $3.74 \mathrm{eV}$. We conclude that interstitial diffusion of $\mathrm{Be}$ has a high barrier in zinc-blende GaN.

\section{Interstitial $\mathrm{Be}\left(\mathrm{Be}_{\text {int }}\right)$ in wurtzite $\mathrm{GaN}$}

The formation energy of the interstitial species $\mathrm{Be}_{i n t}^{2+}$ is shown in Fig. 3 and listed in Table I. Figure 3 indicates that the formation energy of the interstitial donor is low enough to be a serious concern for compensation. We already pointed out that this concern is even more severe in the zincblende structure, where the formation energy of the interstitial is $0.61 \mathrm{eV}$ lower.

When trying to grow $p$-type GaN doped with Be acceptors, one should thus be aware of potential compensation by Be interstitials. The extent to which interstitials form a problem, as well as the extent to which their incorporation can be controlled, depend sensitively on their diffusivity. The fact that Be interstitials can be readily incorporated is due to the small atomic size of the Be atom; one might therefore suspect that this small atom could also diffuse readily through the lattice.

\section{Total-energy surfaces}

To study the behavior of the Be interstitial in GaN, we have calculated the total energy of $\mathrm{Be}_{i n t}$ at various locations in $\mathrm{GaN}$. For each position of Be, the surrounding host atoms are allowed to relax. The resulting energy values as a function of the coordinates of the Be position, $\mathbf{R}_{\mathrm{Be}}$, define the energy surface: $E=E\left(\mathbf{R}_{\mathrm{Be}}\right)$. The energy surface is therefore a function of three spatial dimensions. In order to obtain accurate results, a large data base of energy values (for a large number of spatial coordinates) is needed. In order to render the computational effort tractable, we carried out these calculations in 32-atom wurtzite cells with one $k$ point. As discussed in Sec. II C, this affects energy differences between different Be sites by less than $0.4 \mathrm{eV}$. This accuracy is sufficient not to affect the qualitative shape of the energy surface. Local minima and saddle points identified in this investigation were subsequently also calculated using the 96atom supercell.

We calculated 59 different locations in the irreducible portion of the wurtzite unit cell. These locations were not equally spaced in the crystal, because we chose to calculate more points near the important locations (the local minima and saddle points). For each local minimum, we also allowed $\mathrm{Be}_{i n t}$ to relax to ensure that the actual minimum energy and lattice structure were obtained.

In order to analyze and visualize the total-energy surface, we want to make optimal use of symmetry information. ${ }^{42}$ Since the energy surface is a function of $\mathbf{R}_{\mathrm{Be}}$, it possesses the full symmetry of the crystal. To make effective use of these symmetries, an analytic description of the surface is essential. We achieve this through expansion in a basis set with the appropriate symmetry. We then perform a fitting of the total-energy results to this set of basis functions using a least-squares method. As basis functions, we use symmetrized plane waves that reflect the full symmetry of the wurtzite crystal. ${ }^{42}$ We also make use of the physical fact that 

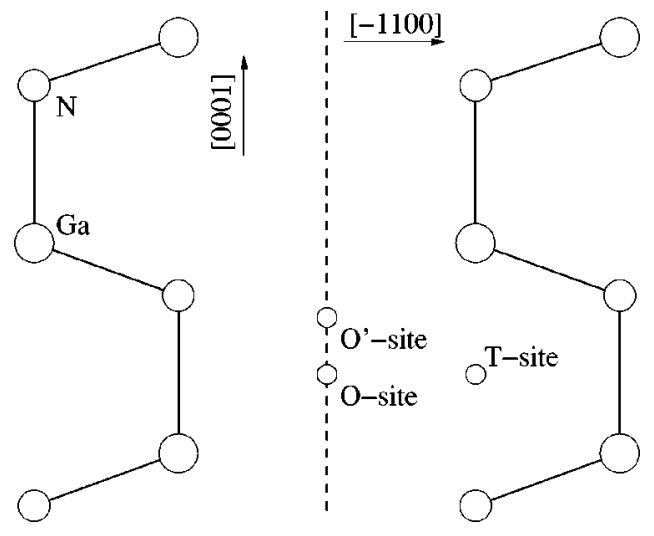

FIG. 5. Schematic representation of atomic positions in the $(11 \overline{2} 0)$ plane of wurtzite GaN. The large circles represent Ga atoms, medium circles $\mathrm{N}$ atoms. The high-symmetry interstitial sites are indicated: $O$ is the octahedral interstitial site and $T$ the tetrahedral interstitial site. $O^{\prime}$ indicates the position of the global minimum for $\mathrm{Be}_{i n t}^{2+}$.

the Be impurity can never approach any host atom too closely (exchange processes, which would carry a very high energy cost, are not included in the total-energy surface). To this end, we add some ad hoc high-energy values near the host atoms to the calculated data set. This addition does not affect the shape of the energy surface in the relevant regions away from the atoms.

Since the energy surface is a function of three spatial dimensions, it is difficult to present the results in a single plot. For visualization purposes, we need to show a cut of the energy surface, restricting the Be coordinates to a single plane. Judicious choice of such planes ensures that we convey all the essential information, i.e., stable and metastable sites as well as barriers between them. For the system con-

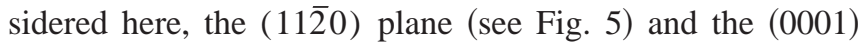
plane (perpendicular to the $c$ axis) are the relevant planes. The energy surface can then be displayed as a contour plot or as a perspective plot of the energy (along the $z$ axis) as a function of coordinates in the plane.

In the wurtzite structure, there are two distinct types of interstitial open space, as shown in Fig 5. $T$ is the tetrahedral interstititial site (or cage site). This site is equidistant from four $\mathrm{Ga}$ and four $\mathrm{N}$ atoms. $O$ is the octahedral interstitial site (or tunnel site). This site is in the "interstitial channel", along the $c$ axis. The $O$ site is equidistant from six $\mathrm{Ga}$ and six $\mathrm{N}$ atoms. Both sites are obvious candidates for local minima (or, more generally, extrema) for $\mathrm{Be}_{i n t}$.

\section{2. $B e_{\text {int }}$ near the $O$ site}

Placing the Be interstitial at the $O$ site and allowing all atoms to relax results in a position (which we call the $O^{\prime}$ site) where Be optimizes its distance to its $\mathrm{N}$ neighbors. This position constitutes the global minimum in the total-energy surface for $\mathrm{Be}_{i n t}^{2+}$ in $\mathrm{GaN}$. As can be seen in Fig. 6(a), the Be atom stays along the axis of the open channel, and moves close to the plane of the $\mathrm{N}$ atoms. The resulting $\mathrm{Be}-\mathrm{N}$ dis- tance $(1.59 \AA)$ is close to the optimum Be-N bond length as observed in, say, $\mathrm{Be}_{3} \mathrm{~N}_{2}$. In the process, the $\mathrm{N}$ atoms move toward Be by $0.22 \AA$.

The formation energy at this global minimum is $0.61 \mathrm{eV}$ higher than for Be in $\mathrm{ZB} \mathrm{GaN} \mathrm{(see} \mathrm{Table} \mathrm{I).} \mathrm{One} \mathrm{might} \mathrm{think}$ that the cause for the higher formation energy in wurtzite is the smaller size of the interstitial cage. However, the fact that for $\mathrm{Be}_{i n t}$ at the $O^{\prime}$ site the nitrogen neighbors actually move toward the interstitial indicates that available volume is not an issue. The Be atom clearly likes to bind to the $\mathrm{N}$ atoms; in the open channel of the WZ structure, it can only effectively form bonds with three $\mathrm{N}$ atoms. At the $T_{d}^{\mathrm{N}}$ site in $\mathrm{ZB}$, on the other hand, it can strongly bind to four $\mathrm{N}$ neighbors. We suggest that this is the reason for the lower formation energy in zinc-blende $\mathrm{GaN}$. We also find that the bonding between the $\mathrm{Be}$ and $\mathrm{N}$ atoms exhibits a significant covalent component, in addition to the ionic component; this emerges from an inspection of the charge density corresponding to various eigenstates throughout the valence band.

For this global minimum, we have also examined other charge states. The Be interstitial does not introduce any Kohn-Sham states within the LDA band gap, indicating that it behaves as a shallow donor. We can calculate the formation energy of the +1 and 0 charge states by placing one or two electrons in the lowest unoccupied level. As for the case of shallow acceptors, the formation energies need to be corrected with a term $E_{\text {corr }}$ reflecting the energy difference between electrons at the special point and at the $\Gamma$ point, as discussed in Sec. II D. In addition, these formation energies suffer from the LDA band gap error; however, if we focus on energy differences between charge states, as appropriate for the calculation of ionization energies, then the results do not directly depend on the LDA error if we express the ionization level with respect to the conduction-band minimum.

Following this procedure, we find the $+/ 0$ transition to correspond to an effective-mass state, while the $++/+$ level is slightly deeper, namely, $0.09 \mathrm{eV}$ below the conduction band. We will revisit this result in Sec. V B.

Coming back to $\mathrm{Be}_{i n t}^{2+}$, the features of the energy surface in the neighborhood of the $O^{\prime}$ site can be obtained from Fig. 7. This figure includes a contour plot [Fig. 7(a)] and a perspective plot [Fig. 7(b)] for the (11520) plane that cuts through the hexagonal channel. We discuss these plots in more detail in the following sections.

\section{3. $B e_{\text {int }}$ at the $T$ site}

We place $\mathrm{Be}_{i n t}$ at the ideal $T$ site and allow the host atoms to relax. If we allow $\mathrm{Be}_{\text {int }}$ to relax along [0001], we find that it stays almost at the ideal position of the $T$ site; we therefore do not need to introduce a separate notation for the minimum-energy site, as we did in the case of the $O^{\prime}$ site. The $T$ site is thus a local minimum for displacements parallel to [0001]. However, when we allow displacements perpendicular to [0001] (and move Be slightly off this axis, in order to break the symmetry), we find that the $T$ site is a local maximum in the plane perpendicular to [0001]. We conclude that $T$ is actually a saddle point. A contour plot and a per-

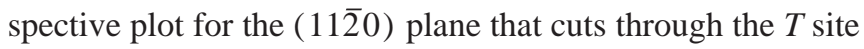




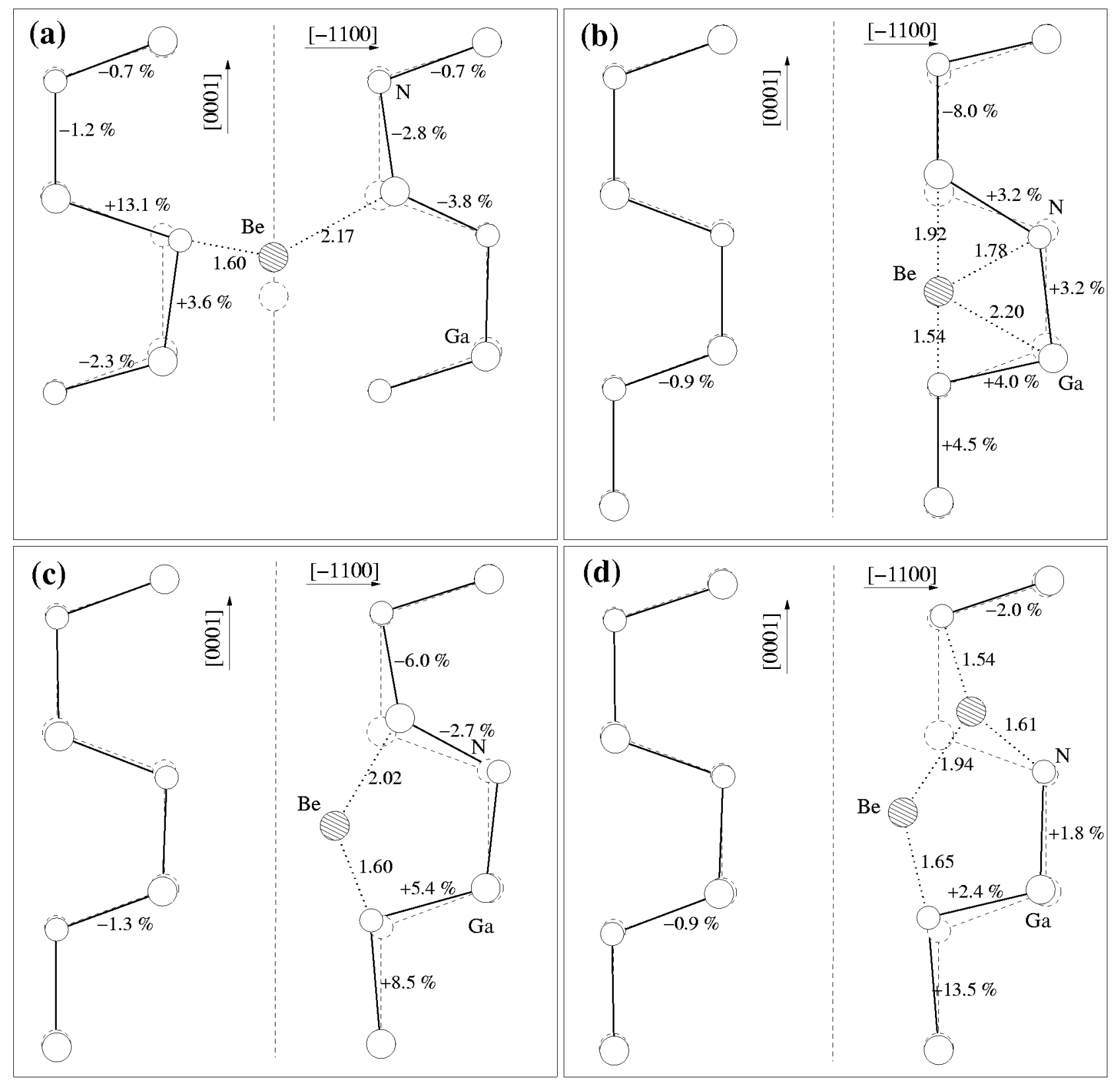

FIG. 6. Schematic representation of atomic positions in the $(11 \overline{2} 0)$ plane for $\mathrm{Be}_{i n t}^{2+}$ at various interstitial sites in wurtzite GaN. Large circles represent $\mathrm{Ga}$ atoms, medium circles $\mathrm{N}$ atoms, and the hatched circle represents Be. Dashed circles indicate ideal atomic positions, dashed lines bonds in the ideal lattice. Distances between Be and its neighbors are given in $\AA$ and changes in Ga-N bond lengths are given as the percentage change from the bulk Ga-N bond lengths. The panels correspond to a Be interstitial in the following configurations: (a) $O^{\prime}$ site; (b) $T$ site; (c) $A$ site; and (d) the $\mathrm{Be}_{\mathrm{Ga}}-\mathrm{Be}_{i n t}^{+}$complex.

are shown in Figs. 7(a) and 7(b), respectively. The corresponding plots for the (0001) plane that cuts through the $T$ site are shown in Figs. 7(c) and 7(d). Figure 7(d) clearly shows that $T$ is a local maximum in the (0001) plane. In the (112̄0) plane, the $T$ site is a local minimum in the [0001] direction and a local maximum in the $[-1100]$ direction.

The position of the $\mathrm{Be}$ atom and the relaxations of the host atoms are shown in Fig. 6(b). Although the host atoms undergo quite large relaxations, the $\mathrm{Be}$ atom is squeezed rather tightly between the $\mathrm{Ga}$ and $\mathrm{N}$ atoms along the $c$ axis, with a Be-Ga distance of $1.92 \AA$ and a Be-N distance of 1.54 $\AA$. In spite of this tight fit, the $T$ site is only $1.18 \mathrm{eV}$ higher in energy than the global minimum at the $O^{\prime}$ site (as calculated in the 96-atom supercell; in the 32-atom cell, the energy difference is $0.90 \mathrm{eV}$ ). The energy at $T$ is probably lowered due to the fact that the Be atom can now bond with four $\mathrm{N}$ neighbors; but the size constraints, in particular the repulsion with the $\mathrm{Ga}$ atom along the $c$ axis, raise the energy.

\section{Be $e_{\text {int }}$ at the $A$ site}

In the process of exploring the total-energy surface for $\mathrm{Be}_{\text {int }}$, we discovered an additional local minimum. Figures 7(a) and 7(b) show a contour plot and a perspective plot for 

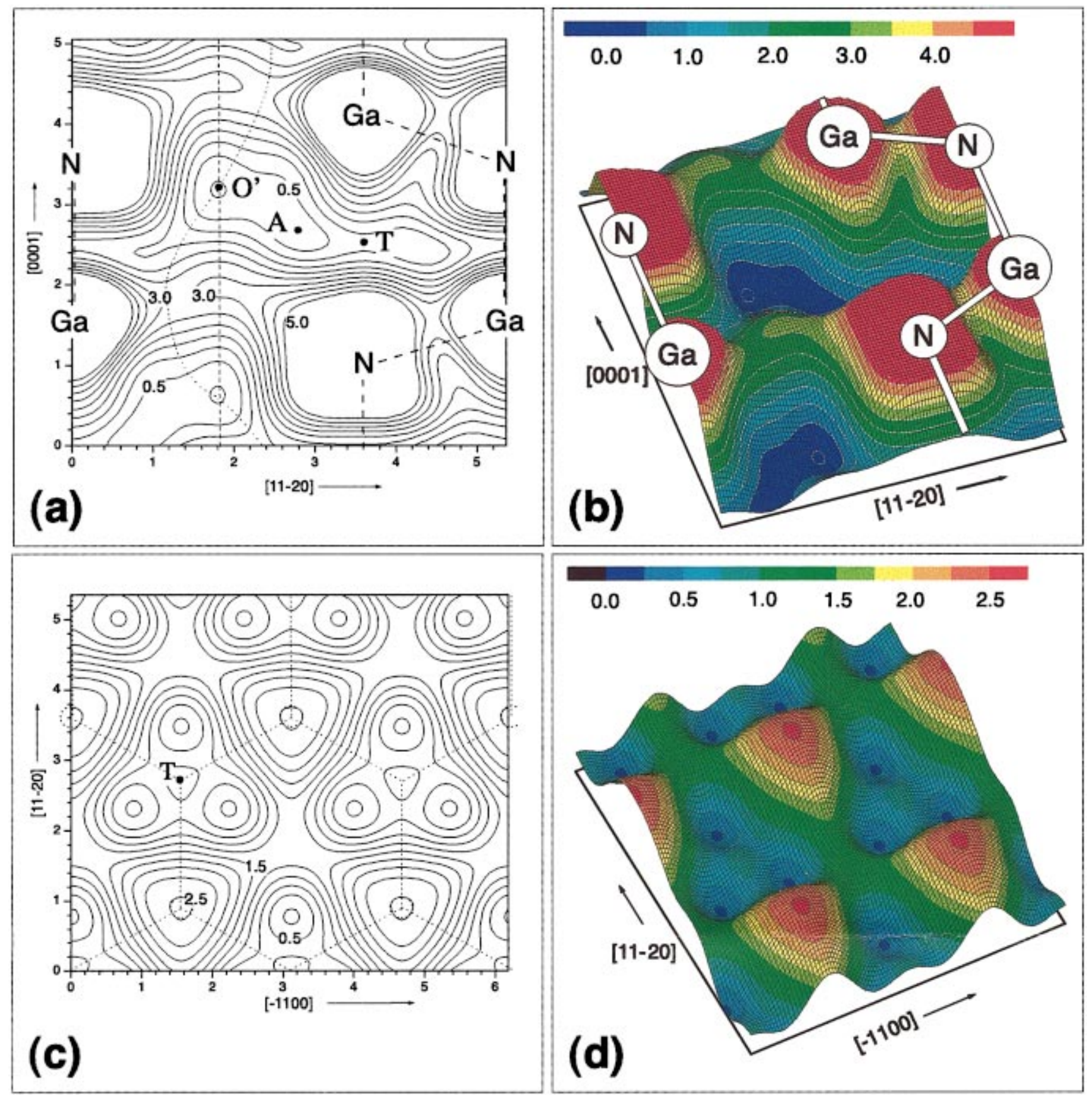

FIG. 7. (Color) Calculated total-energy surfaces for Be interstitials in GaN. (a) Contour plot and (b) perspective plot of the total-energy surface for $\mathrm{Be}_{i n t}^{2+}$ in a $(11 \overline{2} 0$ ) plane through the host atoms (as defined in Fig. 5). In the contour plot, the interval is $0.5 \mathrm{eV}$ and the real-space dimensions along both directions are in $\AA$. (c) Contour plot and (d) perspective plot of the total-energy surface for Be int $^{2+}$ in a (0001) plane through the $T$ site. This (0001) plane thus also cuts through the middle of Ga-N bonds along the $c$ axis. In the contour plot, the interval is $0.25 \mathrm{eV}$ and the real-space dimensions along both directions are in $\AA$. Dashed circles show the projections of the atomic positions of the host atoms and dashed lines show the projections of Ga-N bonds.

the (112̄0) plane that cuts through both $T$ site and $O$ site. We notice that there is a local minimum located between the $T$ site and the $O^{\prime}$ site; we will call this site the $A$ site. The atomic positions for $\mathrm{Be}_{i n t}$ at the $A$ site are shown in Fig. 6(c). This local minimum has a formation energy only $0.70 \mathrm{eV}$ higher than the global minimum (as calculated in the 96- atom supercell; in the 32-atom cell, the energy difference is $0.30 \mathrm{eV}$ ). Again, the driving force seems to be the formation of Be-N bonds; two of the $\mathrm{N}$ atoms move toward $\mathrm{Be}$ by $0.19 \AA$, while a third moves by $0.08 \AA$. This results in three Be-N bonds with bond lengths of $1.60 \AA$ A. Simultaneously, a $\mathrm{Ga}$ atom that would prevent $\mathrm{Be}$ from approaching $\mathrm{N}$ moves 


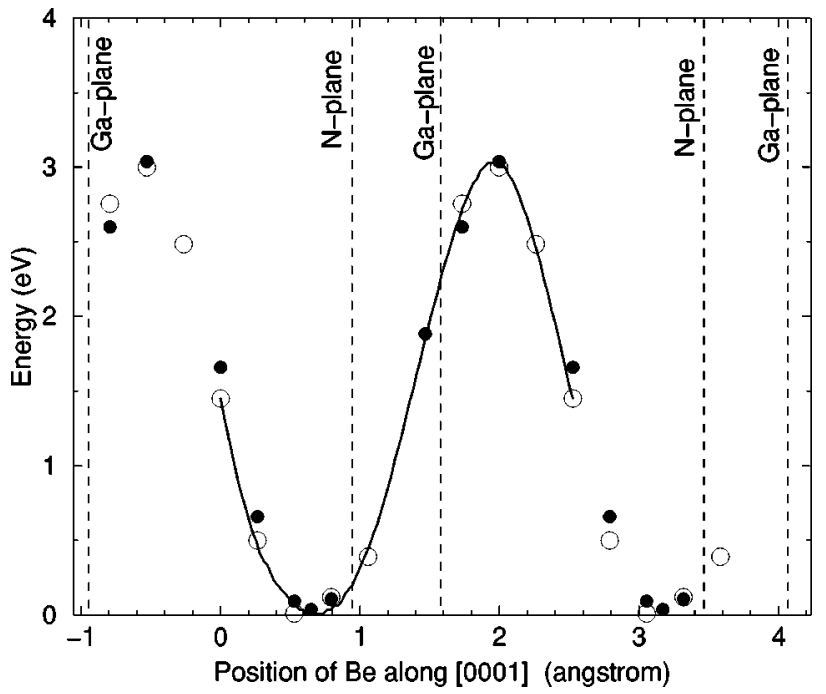

FIG. 8. Total energy of $\mathrm{Be}_{i n t}^{2+}$ at positions along the center of the open channel in WZ GaN, measured from the $O$ site, and referenced to the energy at the global minimum $\left(O^{\prime}\right)$. Solid circles: the results from $1 \times 1 \times 1 k$-mesh calculations; open circles: $2 \times 2 \times 2 \mathrm{k}$-mesh calculations; solid line: interpolation curve.

away, undergoing a displacement of almost $20 \%$ of the Ga-N bond length. Still, the resulting Be-Ga distance of $2.02 \AA$ is probably the determining factor limiting the stability of this site.

\section{Diffusion path for $B e_{i n t}$}

The total-energy surfaces allow us to study the diffusion path of the $\mathrm{Be}$ interstitial along different directions. We focused on the +2 charge state, which is the relevant charge state for $\mathrm{Be}_{i n t}$ at its global minimum (see Sec. III C 2). Inspection of the electronic structure for various positions of Be along its migration path indicates that there is no tendency for localized electronic levels to move into the band gap, and hence no possibility for charge-state changes to occur.

To study the diffusion path along the [0001] direction, we first calculated the formation energies for $\mathrm{Be}_{i n t}$ at several positions along a [0001] direction through an $O$ site (i.e., along the center of the open channel). The results are shown in Fig. 8. This diffusion path yields a diffusion barrier of 3.1 $\mathrm{eV}$ (in the 32-atom cell, very close to the $3.0 \mathrm{eV}$ obtained in a 96-atom cell). Inspection of the full total-energy surface, as shown in Fig. 7, indicates that the lowest-energy path does not run exactly along the center of the channel. The dashed line shows the diffusion path along the center of the channel, while the dotted line shows the actual path; the barrier along the latter path is only slightly lower, at $2.90 \mathrm{eV}$.

For diffusion in a direction perpendicular to the $c$ axis, the interstitial travels between $O^{\prime}$ sites in adjacent channels via a path that has a saddle point at the $T$ site. The diffusion barrier is thus equal to the energy difference between the $T$ and $O^{\prime}$ sites, which is $1.18 \mathrm{eV}$ (in the 96-atom cell; $0.90 \mathrm{eV}$ in the 32-atom cell).
We thus find a very large difference in barrier heights for diffusion parallel to [0001] vs perpendicular to [0001]. A rough estimate of the temperature at which the interstitials would be mobile can be obtained by taking the usual definition of an activation temperature, i.e., the temperature at which the jump rate is one per second, and assuming a prefactor of $10^{13} \mathrm{~s}^{-1}$, i.e., a typical phonon frequency. With a $1.18 \mathrm{eV}$ barrier, the Be interstitial should be mobile in directions perpendicular to the $c$ axis at temperatures of about $180{ }^{\circ} \mathrm{C}$. Diffusion along the $c$ axis, on the other hand, with a barrier of $2.90 \mathrm{eV}$, would require temperatures of about $850{ }^{\circ} \mathrm{C}$

The large anisotropy of the diffusion barriers can be attributed to the features of Be bonding with the host atoms that we described above: $\mathrm{Be}_{i n t}^{2+}$ prefers to be close to at least three $\mathrm{N}$ atoms, with a bond length comparable to that in $\mathrm{Be}_{3} \mathrm{~N}_{2}$. At the same time, $\mathrm{Be}_{i n t}^{2+}$ tries to stay as far away as possible from the $\mathrm{Ga}$ atoms, with which it has a repulsive interaction. As can be seen from Fig. 8, the saddle point for migration along [0001] occurs close to the plane of the $\mathrm{Ga}$ atoms. These atoms form part of a hexagonal ring in the "chair' configuration; in this configuration, the center of the triangle formed by the three $\mathrm{N}$ atoms is displaced from the center of the triangle formed by the Ga atoms by $\sim 0.6 \AA$ along the $c$ axis. Because of the Be-Ga repulsion, squeezing through the triangle of $\mathrm{Ga}$ atoms carries a large energy cost. Simultaneously, the Be atom is far away from the $\mathrm{N}$ atoms with which it prefers to bond. These features conspire to create a large barrier for Be motion along the $c$ axis.

For motion perpendicular to the $c$ axis, the Be atom also has to squeeze through hexagonal rings, but this time these rings have the "boat' configuration. In this configuration, the center of the triangle formed by the $\mathrm{N}$ atoms coincides with the center of the triangle formed by the Ga atoms. This configuration thus allows the Be to stay more closely bonded to the $\mathrm{N}$ atoms, even while squeezing through the triangle of $\mathrm{Ga}$ atoms, resulting in a lower barrier.

\section{RESULTS FOR COMPLEXES}

\section{A. Complexes between interstitial Be and substitutional Be $\left(\mathrm{Be}_{i n t}-\mathrm{Be}_{\mathrm{Ga}}\right)$ in wurtzite $\mathrm{GaN}$}

Since interstitial $\mathrm{Be}$ is a donor $\left(\mathrm{Be}_{i n t}^{2+}\right)$, we expect it will bind with substitutional $\mathrm{Be}$, which is an acceptor $\left(\mathrm{Be}_{\mathrm{Ga}}^{-}\right)$. We explored various possible atomic configurations for the complex; the final stable geometry is illustrated in Fig. 6(d). We notice a similarity with the $A$ site configuration of the $\mathrm{Be}$ interstitial, ilustrated in Fig. 6(c). Indeed, although this metastable site for $\mathrm{Be}_{\text {int }}^{2+}$ was somewhat higher in energy than the $O^{\prime}$ site, the Be interstitial is quite close to a substitutional $\mathrm{Ga}$ site in this $A$ site configuration. This offers the possibility for forming a strong bond to a $\mathrm{Be}_{\mathrm{Ga}}$ substituting on this site.

The relaxation can be described as follows. $\mathrm{Be}_{i n t}$ moves toward $\mathrm{Be}_{\mathrm{Ga}}$ while $\mathrm{Be}_{\mathrm{Ga}}$ moves away slightly in the opposite direction. The two Be atoms form a bond with a bond length of $1.94 \AA$. An alternative way of looking at this configuration is as a split interstitial, with the two Be atoms sharing the substitutional $\mathrm{Ga}$ site; the center of the $\mathrm{Be}-\mathrm{Be}$ bond is displaced $0.46 \AA$ from the ideal Ga position. Both Be atoms 
TABLE III. Calculated binding energies $E^{b}$ [Eq. (13)] and lattice relaxations for various $\mathrm{Be}-\mathrm{H}$ complexes in wurtzite $(\mathrm{WZ})$ and zinc-blende (ZB) GaN. AB stands for antibonding, BC for bondcenter sites. For wurtzite, the symbol $\|$ denotes configurations with the $\mathrm{H}-\mathrm{N}$ bond parallel to the $c$ axis ([0001]); the other bond directions are denoted by the symbol $\perp . \Delta d_{\mathrm{Be}(\mathrm{N})}$ denotes the displacement of the $\mathrm{Be}(\mathrm{N})$ atom from its nominal lattice site, expressed as a percentage of the bond length. $d_{\mathrm{N}-\mathrm{H}}$ denotes the $\mathrm{N}-\mathrm{H}$ distance.

\begin{tabular}{ccccc}
\hline \hline Configuration & $E^{b}(\mathrm{eV})$ & $\Delta d_{\mathrm{Be}}$ & $\Delta d_{\mathrm{N}}$ & $d_{\mathrm{N}-\mathrm{H}}(\AA)$ \\
\hline $\mathrm{WZ} \mathrm{BC}_{\|}$ & 1.81 & $35.3 \%$ & $10.8 \%$ & 1.028 \\
$\mathrm{WZ}, \mathrm{BC}_{\perp}$ & 1.68 & $31.3 \%$ & $10.6 \%$ & 1.027 \\
$\mathrm{WZ}, \mathrm{AB}_{\perp}$ & 0.95 & $21.2 \%$ & $12.8 \%$ & 1.031 \\
$\mathrm{WZ}, \mathrm{AB}_{\|}$ & 0.69 & $23.3 \%$ & $11.3 \%$ & 1.030 \\
$\mathrm{ZB}, \mathrm{BC}$ & 1.64 & $30.4 \%$ & $13.1 \%$ & 1.026 \\
$\mathrm{ZB}, \mathrm{AB}$ & 0.90 & $21.8 \%$ & $13.9 \%$ & 1.032 \\
\hline \hline
\end{tabular}

actually succeed in forming strong bonds with $\mathrm{N}$ atoms: the $\mathrm{Be}$ atom closest to the substitutional site binds with two $\mathrm{N}$ atoms, one at $1.54 \AA$, the other at $1.61 \AA$, while the Be closer to the interstitial site binds with one $\mathrm{N}$ at $1.65 \AA$ and two $\mathrm{N}$ [out of the plane in Fig. 6(d)] at $1.61 \AA$. The Be-Be bond lies

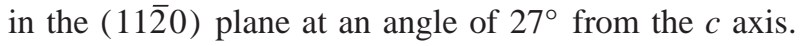

The formation energy of this complex at $E_{F}=0$, calculated using Eq. (5), is $0.37 \mathrm{eV}$ for N-rich conditions (see Table I). The binding energy between $\mathrm{Be}_{\text {int }}^{2+}$ and $\mathrm{Be}_{\mathrm{Ga}}^{-}$is thus calculated to be $1.35 \mathrm{eV}$. The formation energy of the complex as a function of $E_{F}$ is also included in Fig. 3 .

We have also investigated the neutral charge state of this complex. No Kohn-Sham states were introduced within the LDA band gap, indicating a shallow donor. We followed the same procedure as for $\mathrm{Be}_{i n t}$ (see Sec. III C 2) to derive the donor ionization energy, resulting in a level about $0.02 \mathrm{eV}$ below the conduction-band minimum.

If $\mathrm{Be}_{i n t}-\mathrm{Be}_{\mathrm{Ga}}$ are incorporated in bulk $\mathrm{GaN}$, one may wonder whether it is possible to subsequently dissociate them. An estimate for the dissociation energy can be obtained by adding the activation energy for interstitial diffusion to the binding energy of the complex. We are allowed to use the lower of the calculated interstitial diffusion barriers, since for

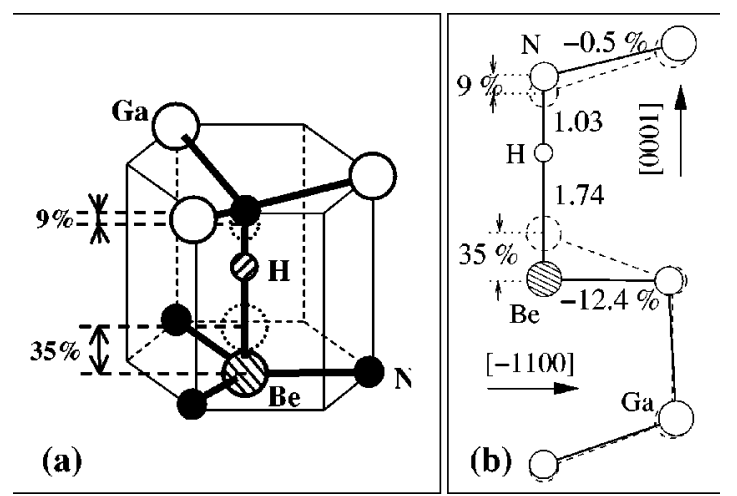

FIG. 9. Schematic illustration of the atomic structure of the $\mathrm{Be}-\mathrm{H}$ complex. The $\mathrm{H}$ atom is located in a bond-center site along the $c$ axis. The $\mathrm{Be}_{\mathrm{Ga}}^{-}$is relaxed outward by a very large amount, moving it into the plane of its three $\mathrm{N}$ neighbors.
TABLE IV. Calculated formation energies for complexes between Be substitutional acceptors $\left(\mathrm{Be}_{\mathrm{Ga}}\right)$ and oxygen substitutional donors $\left(\mathrm{O}_{\mathrm{N}}\right)$ in wurtzite $(\mathrm{WZ})$ and zinc-blende (ZB) GaN. Nitrogen-rich conditions are assumed, as well as equilibrium with $\mathrm{Be}_{3} \mathrm{~N}_{2}$ and $\mathrm{BeO}$. The formation energies are given for $E_{F}=0$, i.e., $E_{F}$ located at the top of the valence band.

\begin{tabular}{lcc}
\hline \hline & \multicolumn{2}{c}{ Formation energies $(\mathrm{eV})$} \\
& $\mathrm{WZ}$ & $\mathrm{ZB}$ \\
\hline$\left(\mathrm{O}_{\mathrm{N}}\right)^{+}$ & 0.24 & 0.22 \\
$\left(\mathrm{Be}_{\mathrm{Ga}}-\mathrm{O}_{\mathrm{N}}\right)^{0}$ & 0.59 & 0.66 \\
$\left(\mathrm{Be}_{\mathrm{Ga}}-\mathrm{O}_{\mathrm{N}}-\mathrm{Be}_{\mathrm{Ga}}\right)^{-}$ & 1.97 & 1.93 \\
$\left(\mathrm{Be}_{\mathrm{Ga}}-\mathrm{O}_{\mathrm{N}}-\mathrm{Be}_{\mathrm{Ga}}\right)^{0}$ & 1.83 & 1.78 \\
\hline \hline
\end{tabular}

purposes of breaking the complex the Be interstitial would presumably choose to move in the direction that offers the lowest dissociation barrier. This yields a dissociation energy of $1.35+1.18=2.53 \mathrm{eV}$. We will discuss in Sec. V what choice of annealing temperatures would result in dissociation of these complexes.

\section{B. Beryllium-hydrogen complexes}

Hydrogen is known to passivate dopant impurities in semiconductors, and hydrogen passivation of $\mathrm{Mg}$ acceptors in $\mathrm{GaN}$ has been well documented..$^{21-23}$ In the case of $\mathrm{Mg}$, the (electrically neutral) $\mathrm{Mg}-\mathrm{H}$ complex was found to have a binding energy of $0.7 \mathrm{eV}$, with the $\mathrm{H}$ atom located in an antibonding $(\mathrm{AB})$ site behind a $\mathrm{N}$ neighbor of the acceptor. ${ }^{22}$

We have carried out comprehensive calculations of the Be-H complex, the results of which are summarized in Table III. The binding energy of the complex is defined with respect to an isolated $\mathrm{Be}$ acceptor in the negative charge state, and an isolated interstitial $\mathrm{H}$ atom in the positive charge state (its lowest-energy state in $p$-type $\mathrm{GaN}$ ) far away from the $\mathrm{Be}$ acceptor:

$$
E_{b}(\mathrm{Be}-\mathrm{H})=-E^{f}\left[\mathrm{Be}-\mathrm{H}^{0}\right]+E^{f}\left[\mathrm{Be}_{\mathrm{Ga}}^{-}\right]+E^{f}\left[\mathrm{H}^{+}\right],
$$

where the signs have been chosen such that a positive binding energy corresponds to a stable complex.

In the wurtzite structure, the $\mathrm{BC}_{\|}$configuration, in which the $\mathrm{H}$ atom is located in the bond-center (BC) site between the $\mathrm{Be}$ acceptor and its $\mathrm{N}$ neighbor along the $c$ axis, is most favorable (highest binding energy). This configuration is illustrated in Fig. 9. The other possible BC configuration, $\mathrm{BC}_{\perp}$, in which the Be-H-N bond is pointing along a direction other than [0001], is only $0.1 \mathrm{eV}$ higher in energy. Note that the $\mathrm{AB}$ configurations are significantly higher in energy than the $\mathrm{BC}$ structures (by more than $0.8 \mathrm{eV}$ ). This result is markedly different from the case of $\mathrm{Mg}-\mathrm{H}$, where the $\mathrm{AB}$ configuration was the more favorable one. Our calculated binding energy for the global minimum of the Be-H complex is in good agreement with that reported by Bernardini et al. ${ }^{6}$

We also draw attention to the extremely large atomic relaxations in this complex: the $\mathrm{N}$ atom moves outward by about $10 \%$ of the Ga-N bond length, and the Be by as much as $35 \%$. Such large relaxations were actually also calculated for the Mg-H complex. 


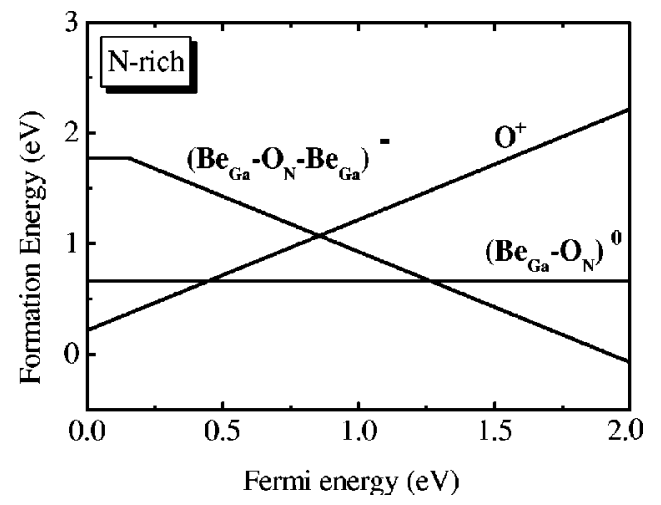

FIG. 10. Calculated defect formation energies as a function of Fermi level for complexes between oxygen and beryllium in zincblende $\mathrm{GaN}$, under N-rich conditions.

For all the complexes listed in Table III, the N-H distance is approximately $1.03 \AA$. Also note that the behavior of the complexes in the zinc-blende phase is qualitatively very similar to that in the wurtzite phase: The BC configuration is significantly more stable than the $\mathrm{AB}$ configuration, and the atomic relaxations (particularly of the Be impurity) are very large.

Experimental identification of acceptor-hydrogen complexes often relies on vibrational spectroscopy. We have therefore also calculated the vibrational modes of the Be-H complex, for the most favorable geometry of the complex (i.e., $\mathrm{BC}_{\|}$). In the harmonic approximation we find a stretch mode at $3460 \mathrm{~cm}^{-1}$, and a wag mode at $570 \mathrm{~cm}^{-1}$. As expected, the stretch mode is representative of a N-H bond, which dominates the bonding in this complex, while the wag modes are low in frequency because the potential energy surface perpendicular to the bond is quite shallow for the BC configuration. ${ }^{42}$ More details about the vibrational mode calculation will be published elsewhere. ${ }^{43}$

\section{Beryllium-oxygen and Be-O-Be complexes}

Complex formation between $\mathrm{Be}_{\mathrm{Ga}}$ and oxygen has been suggested as an explanation for the high hole conductivities observed in codoping experiments. ${ }^{18-20}$ We have carried out calculations for a variety of complexes between oxygen and one or two Be atoms. The results are summarized in Table IV and Fig. 10. In Fig. 10 we focus on zinc blende, which is the phase for which experimental results were reported; ${ }^{18}$ however, we notice from Table IV that the results for wurtzite are very similar. The choice of chemical potentials was as follows. We chose N-rich conditions, to allow easy comparison with results presented elsewhere in this paper. For Be, we again assumed its chemical potential to be limited by equilibrium with $\mathrm{Be}_{3} \mathrm{~N}_{2}$. For oxygen, finally, we assumed equilibrium with $\mathrm{BeO}$ (for which we used the experimental heat of formation $-6.3 \mathrm{eV}$; Ref. 37). These specific choices are not critical for the qualitative conclusions that we will derive. Note, however, that if we did not include equilibrium with $\mathrm{BeO}$ the formation energies of any of the oxygencontaining complexes would be much lower; indeed, the high binding energy of the Be-O bond favors formation of such complexes.
First we examine the $\left(\mathrm{Be}_{\mathrm{Ga}}-\mathrm{O}_{\mathrm{N}}\right)^{0}$ complex, which is neutral because the $\mathrm{O}_{\mathrm{N}}^{+}$donor passivates the $\mathrm{Be}_{\mathrm{Ga}}^{-}$acceptor. The binding energy of the complex is defined with respect to an isolated $\mathrm{Be}$ acceptor in the negative charge state, and an isolated $\mathrm{O}$ donor in the positive charge state far away from the Be acceptor:

$$
E_{b}\left(\mathrm{Be}_{\mathrm{Ga}}-\mathrm{O}_{\mathrm{N}}\right)=-E^{f}\left[\mathrm{Be}_{\mathrm{Ga}}-\mathrm{O}_{\mathrm{N}}\right]+E^{f}\left[\mathrm{Be}_{\mathrm{Ga}}^{-}\right]+E^{f}\left[\mathrm{O}_{\mathrm{N}}^{+}\right] \text {, }
$$

where the signs have been chosen such that a positive binding energy corresponds to a stable complex. From the calculated formation energies, we obtain a binding energy for $\left(\mathrm{Be}_{\mathrm{Ga}}-\mathrm{O}_{\mathrm{N}}\right)$ of $1.33 \mathrm{eV}$ in $\mathrm{WZ}$ and $1.23 \mathrm{eV}$ in $\mathrm{ZB}$. This is quite a large value, and indicates that these complexes are likely to form.

Interestingly, in this $\mathrm{Be}-\mathrm{O}$ complex the $\mathrm{Be}$ and $\mathrm{O}$ atoms move away from their substitutional lattice sites, in opposite directions. Be moves by a distance equal to $22 \%$ of the Ga-N bond length, and oxygen moves by $12 \%$ of the bond length. The final distance between the $\mathrm{Be}$ and $\mathrm{O}$ atoms is such that only a very weak bond is present between the impurities. We propose the following tentative explanation for the observed tendency for $\mathrm{Be}$ and $\mathrm{O}$ to move away from one another. The natural $\mathrm{Be}-\mathrm{O}$ bond length is very short; for instance, the bond distance in $\mathrm{BeO}$ distance is $1.65 \AA^{44}$ In order to maintain this preferred $\mathrm{Be}-\mathrm{O}$ distance for $\mathrm{Be}$ and $\mathrm{O}$ nearest neighbors in $\mathrm{GaN}$, a large displacement of Be away from its $\mathrm{N}$ neighbors (and/or of $\mathrm{O}$ away from its $\mathrm{Ga}$ neighbors) would be required, in turn leading to significant relaxations of those $\mathrm{N}$ and $\mathrm{Ga}$ atoms. Such large relaxations are energetically costly. Our result indicates that it is actually preferable for $\mathrm{Be}$ and $\mathrm{O}$ to both optimize their bond lengths to their surrounding $\mathrm{N}$ and Ga neighbors, and essentially "give up" on forming a $\mathrm{Be}-\mathrm{O}$ bond. Indeed, both $\mathrm{Be}$ and $\mathrm{O}$ seem to be comfortable when bonded to only three neighbors in $\mathrm{GaN}$; for Be, this tendency has been discussed above in Sec. III A; for $\mathrm{O}$ it has been observed in the context of $D X$-center formation. ${ }^{45}$

Turning now to $\left(\mathrm{Be}_{\mathrm{Ga}}-\mathrm{O}_{\mathrm{N}}-\mathrm{Be}_{\mathrm{Ga}}\right)$ complexes, we note from Table IV and Fig. 10 that these have formation energies that are slightly higher than those of isolated $\mathrm{Be}_{\mathrm{Ga}}$ acceptors. We therefore do not expect a higher solubility of Be based upon formation of such complexes. Our calculated ionization energy of the Be-O-Be complex is $0.14 \mathrm{eV}$ in $\mathrm{WZ}$ and $0.15 \mathrm{eV}$ in $\mathrm{ZB}$; within our error bars, this is comparable to that of the isolated substitutional $\mathrm{Be}$. As to the structure of the $\left(\mathrm{Be}_{\mathrm{Ga}}-\mathrm{O}_{\mathrm{N}}-\mathrm{Be}_{\mathrm{Ga}}\right)$ complex, we again find that one $\mathrm{Be}-\mathrm{O}$ bond is effectively broken. In the WZ structure, the configuration with no Be-O bonds along the $c$ axis was found to be favored. It is informative to calculate the binding energy for the process whereby an additional $\mathrm{Be}_{\mathrm{Ga}}^{-}$is attached to a $\left(\mathrm{Be}_{\mathrm{Ga}}-\mathrm{O}_{\mathrm{N}}\right)$ complex, resulting in a $\left(\mathrm{Be}_{\mathrm{Ga}}-\mathrm{O}_{\mathrm{N}}-\mathrm{Be} \mathrm{Ga}\right)^{-}$complex. Using the tabulated formation energies we find a binding energy of $0.30 \mathrm{eV}$ in $\mathrm{WZ}$ and $0.40 \mathrm{eV}$ in $\mathrm{ZB}$. This binding energy is significantly smaller than the binding energy found above for the Be-O complex. This indicates that once a Be-O complex is formed the driving force for attaching a second $\mathrm{Be}$ atom is quite small. 


\section{DISCUSSION}

\section{A. Incorporation of $\mathrm{Be}$ in $\mathrm{GaN}$}

Our calculated formation energy for $\mathrm{Be}_{\mathrm{Ga}}$ in $\mathrm{GaN}$ is slightly lower than that of $\mathrm{Mg}_{\mathrm{Ga}}$, which is the most widely used acceptor. ${ }^{5,35}$ Comparing our results for formation energies (which determine solubilities) with previous calculations by Bernardini et al., ${ }^{6}$ we find reasonable agreement for the formation energy of the substitutional acceptor (allowing for differences relating to the choice of atomic chemical potentials, etc.). The formation energies obtained in Ref. 6 for interstitial Be are significantly larger than those found in the present work (by more than $2.0 \mathrm{eV}$ ). We are unable to provide an explanation for this discrepancy.

In Sec. III A we pointed out that by moving from N-rich to Ga-rich conditions the formation energy of $\mathrm{Be}_{\mathrm{Ga}}$ increases; indeed, we chose to list results for N-rich conditions precisely because that leads to the highest possible solubility for $\mathrm{Be}_{\mathrm{Ga}}$. It turns out that moving toward Ga-rich conditions also decreases the formation energy of $\mathrm{Be}_{\text {int }}$ (because we are assuming equilibrium with $\mathrm{Be}_{3} \mathrm{~N}_{2}$ ). Optimizing Be incorporation (and suppressing Be interstitial formation) would thus require $\mathrm{N}$-rich conditions. Unfortunately, growing highquality $\mathrm{GaN}$ usually requires Ga-rich growth conditions, ${ }^{46}$ which are less favorable for Be incorporation.

The large binding energies found for $\mathrm{Be}-\mathrm{H}$ complexes (see Table III) are in agreement with the results of Ref. 6. If $\mathrm{H}$ is incorporated during growth, $\mathrm{Be}-\mathrm{H}$ complexes are sure to form. Incorporation of $\mathrm{H}$ may happen unintentionally, such as during MOCVD (metal-organic chemical vapor deposition) growth or when $\mathrm{NH}_{3}$ is used as a source gas in MBE. Intentional exposure to hydrogen may also be obtained by introducing atomic hydrogen in the chamber during growth. The large binding energy of the Be-H complex results in a low formation energy, which would promote incorporation of Be and effectively increase the solubility. We previously discussed this mechanism in the case of $\mathrm{Mg}$ doping. ${ }^{23}$

As in the case of $\mathrm{Mg}$ doping, the resulting acceptorhydrogen complexes need to be broken and the hydrogen removed from the vicinity of the acceptors before electrical activation of the $p$-type layer can be achieved. The binding energy of $1.81 \mathrm{eV}$ may seem to make it difficult to break Be-H complexes. An estimate for the dissociation energy of these complexes can be obtained by adding the activation energy for $\mathrm{H}^{+}$diffusion, $0.7 \mathrm{eV},{ }^{22}$ to the binding energy of the Be-H complex. This yields a dissociation barrier of about $2.51 \mathrm{eV}$. Although this is a high value, it should not be a problem to dissociate the complexes at the temperatures typically used for activation of the $p$-type layers. Indeed, it is well known that hydrogen-acceptor complexes in $\mathrm{Si}$, which have a dissociation energy of about $1.2 \mathrm{eV}$, can be broken up at temperatures of about $150{ }^{\circ} \mathrm{C}$. Assuming similar dissocation kinetics, complexes with a barrier twice as high should therefore dissociate at a temperature of $2 \times(150+273) \mathrm{K}$, or $573{ }^{\circ} \mathrm{C}$. This conclusion is in agreement with the assessment of Bernardini et al. ${ }^{6}$

Experiments on Be incorporation in $\mathrm{GaN}$ are still scarce. Incorporation of $\mathrm{Be}$ in $\mathrm{GaN}$ has so far been performed during MBE growth ${ }^{7-15}$ and using ion implantation. ${ }^{16,17}$ The earliest report of Be doping was by Ilegems and Dingle, ${ }^{47}$ who incorporated Be during vapor growth of $\mathrm{GaN}$. The introduction of Be resulted in a deep yellow-green luminescence band centered around 2.2, which is most likely defect related. Sánchez et al. ${ }^{7-9}$ incorporated $\mathrm{Be}$ in $\mathrm{WZ} \mathrm{GaN}$ grown by MBE on Si substrates. SIMS (secondary ion mass spectroscopy) showed the presence of Be, but due to a lack of calibration no concentrations could be inferred. Conductivity measurements of the Be-doped layer were impossible, due to the electrical activity of $\mathrm{Ga}$ diffused into the underlying $\mathrm{Si}$ substrate. Finally, Cheng et al. ${ }^{10,11}$ reported Be incorporation in $\mathrm{ZB}$ and $\mathrm{WZ}$ GaN grown by MBE on GaAs substrates. SIMS indicated Be incorporation, but again no calibration was available. For high Be concentrations, the SIMS profiles showed evidence of Be diffusion through an undoped GaN buffer layer and into the underlying GaAs substrate.

Ion implantation was used by Ronning et al. ${ }^{16,17}$ to incorporate $\mathrm{Be}$ in MOCVD-grown $\mathrm{GaN}$, followed by annealing at $1100^{\circ} \mathrm{C}$ for at least one hour under an atomic nitrogen flux. Beryllium concentrations of more than $10^{17} \mathrm{~cm}^{-3}$ were obtained as measured by SIMS. No long-range diffusion of Be was observed, but this result could merely reflect the presence of implantation damage.

\section{B. Ionization energies}

Our calculated ionization energy for the Be acceptor in $\mathrm{WZ} \mathrm{GaN}$ is $170 \mathrm{meV}$. This value is slightly lower (by about $30 \mathrm{meV}$ ) than our previously calculated value for $\mathrm{Mg}$ in $\mathrm{GaN}^{23}$ We emphasize that the error bar on these values (which we estimate to be at least $100 \mathrm{meV}$ ) does not allow us to draw firm conclusions about the magnitude of the ionization energy. Still, the similarity of the values for Be and $\mathrm{Mg}$ is in line with the expectation that the ionization energy for these shallow acceptors is largely determined by intrinsic properties of the semiconductor, such as effective masses and dielectric constants. Indeed, predictions from effectivemass theory ${ }^{48}$ for ionization energies of substitutional acceptors in $\mathrm{WZ}$ GaN produce a value for Be between 185 and $233 \mathrm{meV}$ - only slightly lower than the calculated value for $\mathrm{Mg}$.

Our result for the ionization energy is larger than the 60 meV reported by Bernardini et al. ${ }^{6}$ for Be in $\mathrm{WZ} \mathrm{GaN}$. Their value for $\mathrm{Be}$ in $\mathrm{ZB} \mathrm{GaN}$ was even lower, the acceptor level being resonant with the valence band. One potential explanation for the discrepancy is the smaller unit cell (32 atoms) used in their calculations-although our own results for 32atom cells produce ionization energies quite close to the converged values. It is also possible that the correction term $E_{\text {corr }}$, discussed in Sec. II D, was not taken into account. This correction lowers the formation energy of the neutral charge state, and hence increases the ionization energy. Neglect of the correction would thus result in artificially low acceptor ionization energies.

In Sec. III A we reported that the large lattice relaxation for $\mathrm{Be}_{\mathrm{Ga}}^{0}$ would result in a Franck-Condon shift of about 100 $\mathrm{meV}$, this being the energy difference between $\mathrm{Be}_{\mathrm{Ga}}^{-}$with a configuration fixed to be equal to that of the $\mathrm{Be}_{\mathrm{Ga}}^{0}$ neutral acceptor, and $\mathrm{Be}_{\mathrm{Ga}}^{-}$in its lowest-energy, relaxed configura- 
tion (see Fig. 2). We thus expect the optical ionization energy to be higher than the thermal ionization energy by 100 $\mathrm{meV}$ (see Sec. II F). Thermal ionization energies for Be acceptors in $\mathrm{GaN}$ have not yet been reported, due to a lack of electrical conductivity data for Be-doped samples. Photoluminescence spectroscopy has been performed by several groups, however, and various estimates for the optical ionization energy have been published.

Sánchez et al. ${ }^{7,8}$ observed a PL line at $3.384 \mathrm{eV}$, which they attributed to a donor-acceptor transition. This led to an estimate for the optical ionization energy of $90 \mathrm{meV}$. This result agrees with the work of Dewsnip et al. ${ }^{13}$ who reported a PL line at $3.376 \mathrm{eV}$; again assuming this to be a donoracceptor transition, they calculated an ionization energy of 90-100 meV.

Other groups, however, have reached different conclusions regarding the optical ionization energy. Cheng et al. ${ }^{11}$ observed a PL line at $2.90 \mathrm{eV}$ in their zinc-blende material, and estimated an acceptor ionization energy of $200 \mathrm{meV}$. Salvador et al. ${ }^{14}$ observed a PL peak at $390 \mathrm{~nm}(3.187 \mathrm{eV})$, and inferred from this an acceptor ionization energy of 250 $\mathrm{meV}$. They also reported that hot-probe measurements indicated the presence of $p$-type conductivity. Ronning et al. ${ }^{16}$ observed a PL line at $3.35 \mathrm{eV}$ in their Be-implanted material. They attributed the line to band-to-acceptor $(e A)$ transitions, and extracted an ionization energy of $150 \pm 10 \mathrm{meV}$.

The reported values for the optical ionization energy thus cover a wide range, from $90 \mathrm{meV}$ up to $250 \mathrm{meV}$. Based on our calculated Franck-Condon shift of $100 \mathrm{meV}$, we think it is very unlikely that the optical ionization energy of the substitutional acceptor could be as low as $90 \mathrm{meV}$, or even 150 $\mathrm{meV}$. Indeed, this would result in a thermal ionization energy smaller than $50 \mathrm{meV}$, which conflicts with theoretical predictions and for which there is currently no experimental evidence. We suggest that the photoluminescence data producing optical ionization energies below $150 \mathrm{meV}$ be reexamined, in terms of their attribution to an isolated $\mathrm{Be}$ substitutional acceptor and/or the interpretation of the transition responsible for the emission. One could speculate that the observed transition may involve Be interstitials, for which we calculated a $++/+$ ionization level about $90 \mathrm{meV}$ below the conduction band (Sec. III C 2).

\section{Codoping with oxygen}

Brandt et al. ${ }^{18,19}$ reported high levels of Be incorporation in cubic $\mathrm{GaN}$ grown by $\mathrm{MBE}$ on semi-insulating GaN substrates. Oxygen was introduced as a codopant. SIMS indicated concentrations around $5 \times 10^{20} \mathrm{~cm}^{-3}$ for both Be and $\mathrm{O}$. No significant diffusion of $\mathrm{Be}$ into the $\mathrm{GaN}$ buffer layer was observed; formation of $\mathrm{Be}-\mathrm{O}$ complexes was mentioned as a potential reason for the lack of diffusion. Roomtemperature conductivities as high as $50 / \Omega \mathrm{cm}$ were reported. Hall measurements (performed after removal of the substrate) produced hole concentrations of up to $5 \times 10^{18}$ $\mathrm{cm}^{-3}$, and mobilities of up to $150 \mathrm{~cm}^{2} / \mathrm{V} \mathrm{s}$.

Yamamoto and Katayama-Yoshida proposed an explanation for these codoping results in terms of $\mathrm{Be}_{\mathrm{Ga}}-\mathrm{O}_{\mathrm{N}}-\mathrm{Be}_{\mathrm{Ga}}$ complexes. ${ }^{20}$ They performed first-principles calculations, but their arguments were mainly based on trends in the electrostatic (Madelung) energy.

We reported our full energy-minimization calculations for the Be-O-Be complexes in Sec. IV C. We found that the formation energy of these complexes is not lower than that of isolated $\mathrm{Be}$ acceptors (at least if equilibrium with the proper solubility-limiting phases is taken into account). Formation of Be-O complexes (which are electrically neutral) is energetically quite favorable, but attaching a second $\mathrm{Be}$ atom to such a complex is only marginally favored. Finally, even if $\mathrm{Be}-\mathrm{O}-\mathrm{Be}$ complexes form, their ionization energy does not seem to be noticeably lower than that of isolated Be acceptors (within the error bars of our calculations). We therefore doubt that formation of $\mathrm{Be}-\mathrm{O}-\mathrm{Be}$ complexes is a viable approach to increasing $p$-type doping of $\mathrm{GaN}$, or provides an explanation for the experimentally observed $p$-type conductivity in codoped samples.

\section{Compensation and how to avoid it}

Our calculated formation energies for Be interstitials clearly indicate that they would easily incorporate as compensating centers. In WZ GaN, our calculated formation energy of $\mathrm{Be}_{i n t}^{2+}$ is $0.04 \mathrm{eV}+2 E_{F}$. To put this into perspective, this formation energy is lower than that of the nitrogen vacancy (which is the dominating intrinsic defect in $p$-type $\mathrm{GaN}$ ), at least for Fermi-level positions within $1 \mathrm{eV}$ of the top of the valence band.

We note that codoping with hydrogen during growth offers a very effective means of avoiding compensation. Indeed, hydrogen itself is a donor with a very low formation energy, ${ }^{22,23}$ and hence is more likely to incorporate as a compensating center than the Be interstitial—especially when the large binding energy (and hence low formation energy) of the Be-H complex is taken into account. Compensation by Be interstitials would thus be effectively suppressed in the presence of hydrogen. The hydrogen would need to be removed in a postgrowth annealing process before activation of the acceptors could be achieved, but as discussed in Sec $\mathrm{V}$ A that should not pose any problems. We emphasize that the success of codoping crucially depends on the ability to remove the codopant after the layers have been grown. This is possible in the case of hydrogen, but not in the case of other elements (such as oxygen) that have been suggested as codopants. This issue has been discussed in Ref. 23.

If, for any reason, Be interstitials are not incorporated during growth, they might still diffuse into the bulk after growth. Our calculated diffusion barrier for motion along the $c$ axis is $2.90 \mathrm{eV}$; we estimated in Sec. III C 5 that this would result in significant diffusion at temperatures above $850^{\circ} \mathrm{C}$.

Any Be interstitials that are incorporated are likely to bind to $\mathrm{Be}_{\mathrm{Ga}}$ acceptors; indeed, their diffusion barrier (at least perpendicular to $c$ ) is quite low, and $\mathrm{Be}_{i n t}^{2+}$ is electrostatically attracted to $\mathrm{Be}_{\mathrm{Ga}}^{-}$. We found the binding energy of such complexes to be $1.35 \mathrm{eV}$, and in Sec. IV A we estimated the dissociation energy to be $2.53 \mathrm{eV}$. This is very similar to the dissociation energy of Be-H complexes, and thus by the same arguments as used in Sec. V A annealing temperatures 
of $600{ }^{\circ} \mathrm{C}$ or higher should be adequate to break such complexes. The resulting isolated $\mathrm{Be}$ interstitials still need to be "neutralized" in order to achieve activation of the Be acceptors. This can be achieved either by completely removing the Be interstitials from the $p$-type layer, or by neutralizing the interstitial donors, for example at extended defects. If lateral diffusion suffices to achieve this neutralization, then the same temperatures as required for breaking of the complexes should suffice to activate the $p$-type layer. However, if diffusion parallel to $c$ is necessary (for instance, to remove the Be interstitials through the surface of the wurtzite layer), then higher temperatures may be required due to the higher diffusion barrier for interstitials in that direction.

\section{SUMMARY AND OUTLOOK}

We have reported comprehensive first-principles results for $\mathrm{Be}$ in $\mathrm{GaN}$. The formation energy of Be acceptors is slightly lower than that of $\mathrm{Mg}$, indicating that Be would exhibit a higher solubility. We also predict a slightly lower thermal ionization energy for Be. The optical ionization energy is calculated to be higher than the thermal ionization energy by $100 \mathrm{meV}$, due to a large lattice relaxation of the Be acceptor in its neutral charge state.

The calculations show that Be interstitials have a low formation energy, and are hence likely to incorporate during growth. Be interstitials are double donors and hence cause compensation. We mapped out the total-energy surface for diffusion of Be interstitials, finding a highly anisotropic behavior, with diffusion along the $c$ axis having a much larger barrier $(2.9 \mathrm{eV})$ than diffusion in planes perpendicular to the $c$ axis $(1.2 \mathrm{eV})$. The interstitials may bind to substitutional acceptors, with a binding energy of $1.35 \mathrm{eV}$ and a dissociation energy of $2.53 \mathrm{eV}$.

In the presence of hydrogen, beryllium-hydrogen complexes may form with a binding energy of $1.81 \mathrm{eV}$ and a dissociation energy of $2.51 \mathrm{eV}$. Formation of such complexes would result in a higher solubility of substitutional Be acceptors, and suppression of compensation by Be interstitial donors. A postgrowth activation anneal is then required to remove the hydrogen from the vicinity of the Be acceptors.

Based on our results and analysis, we can suggest several experiments that could shed further light on the behavior of $\mathrm{Be}$ in GaN. Obviously, an experimental determination of the thermal ionization energy would be very valuable. Further investigations of the optical ionization energy, particularly in light of the predicted large Franck-Condon shift, are also desired.

To study the diffusion of interstitial $\mathrm{Be}$, diffusion of $\mathrm{Be}$ into GaN from a Be source on the surface could be attempted. For WZ GaN, this would probably mainly involve diffusion along the $c$ axis, which we predicted to have a high barrier. If diffusion along extended defects could be excluded, we would therefore expect only very slow Be diffusion for this experimental configuration. It would be highly interesting if an experimental geometry could be conceived in which interstitial diffusion perpendicular to the $c$ axis could be studied, which we predict to have a relatively low barrier.

If $\mathrm{Be}$ interstitials are incorporated during growth, then postgrowth annealing experiments would be very informative. Indeed, we estimated that typical annealing temperatures of $600{ }^{\circ} \mathrm{C}$ or higher would result in dissociation of $\mathrm{Be}_{i n t}-\mathrm{Be}_{\mathrm{Ga}}$ complexes and rapid in-plane diffusion of $\mathrm{Be}$ interstitials. Diffusion parallel to $c$ may require higher temperatures.

Finally, the role of hydrogen is likely to be a very beneficial one: its intentional or unintentional incorporation should be monitored, formation of Be-H complexes may be detected (for instance by using vibrational spectroscopy and comparing with our predicted vibrational modes), and dissociation of such complexes by thermal annealing should be attempted.

In conclusion, our first-principles studies have elucidated the attractive aspects of beryllium as an acceptor in $\mathrm{GaN}$, but also the potential problems, which are mainly related to the incorporation of Be interstitials. We hope that our comprehensive investigation of the properties of the Be dopant will prove useful in guiding and interpreting experiments.

\section{ACKNOWLEDGMENTS}

This work was supported by the Office of Naval Research, Contract No. N00014-99-C-0161 and by the Air Force Office of Scientific Research, Contract No. F4920-00C-0019. We thank J. E. Northrup, L. Romano, N. Johnson, M. Kneissl, P. Kiesel, and T. H. Myers for useful discussions. C.V.d.W. is grateful to the Fritz-Haber-Institut and Paul-Drude-Institut, Berlin, for their hospitality, and to the Alexander von Humboldt Foundation for financial support.
*Email address: vandewalle@parc.xerox.com

${ }^{1}$ W. Götz, R. S. Kern, C. H. Chen, H. Liu, D. A. Steigerwald, and R. M. Fletcher, Mater. Sci. Eng., B 59, 211 (1999).

${ }^{2}$ D. P. Bour, H. F. Chung, W. Gtz, L. Romano, B. S. Krusor, D. Hofstetter, S. Rudaz, C. P. Kuo, F. A. Ponce, N. M. Johnson, M. G. Craford, and R. D. Bringans, in III-V Nitrides, edited by F. A. Ponce, T. D. Moustakas, I. Akasaki, and B. Monemar, Mater. Res. Soc. Symp. Proc. No. 449 (Materials Research Society, Pittsburgh, 1997), p. 509.

${ }^{3}$ J. Neugebauer and C. G. Van de Walle, in Gallium Nitride and Related Materials, edited by R. D. Dupuis, J. A. Edmond, F. A. Ponce, and S. Nakamura, Mater. Res. Soc. Symp. Proc. No. 395
(Materials Research Society, Pittsburgh, 1996), p. 645.

${ }^{4}$ V. Fiorentini, F. Bernardini, A. Bosin, and D. Vanderbilt, in Proceedings of the 23rd International Conference on the Physics of Semiconductors, edited by M. Scheffler and R. Zimmermann (World Scientific, Singapore, 1996), p. 2877.

${ }^{5}$ J. Neugebauer and C. G. Van de Walle, J. Appl. Phys. 85, 3003 (1999).

${ }^{6}$ F. Bernardini, V. Fiorentini, and A. Bosin, Appl. Phys. Lett. 70, 2990 (1997).

${ }^{7}$ F. J. Sánchez, F. Calle, M. A. Sánchez-García, E. Calleja, E. Muñoz, C. H. Molloy, D. J. Somerford, J. J. Serrano, and J. M. Blanco, Semicond. Sci. Technol. 13, 1130 (1998). 
${ }^{8}$ R. J. Sánchez, F. Calle, M. A. Sánchez-García, E. Calleja, E. Muñoz, C. H. Molloy, D. J. Somerford, F. K. Koschnick, K. Michael, and J.-M. Spaeth, MRS Internet J. Nitride Semicond. Res. 3, 19 (1998).

${ }^{9}$ M. A. Sánchez-García, E. Calleja, F. J. Sánchez, F. Calle, E. Monroy, D. Basak, E. Muñoz, C. Villar, A. Sanz-Hervas, M. Aguilar, J. J. Serrano, and J. M. Blanco, J. Electron. Mater. 27, 276 (1998).

${ }^{10}$ T. S. Cheng, C. T. Foxon, L. C. Jenkins, S. E. Hooper, D. E. Lacklison, J. W. Orton, B. Ya Ber, A. V. Merkulov, and S. V. Novikov, Semicond. Sci. Technol. 11, 538 (1996).

${ }^{11}$ T. S. Cheng, S. E. Hooper, L. C. Jenkins, C. T. Foxon, D. E. Lacklison, J. D. Dewsnip, and J. W. Orton, J. Cryst. Growth 166, 597 (1996).

${ }^{12}$ D. J. Dewsnip, A. V. Andrianov, I. Harrison, D. E. Lacklison, J. W. Orton, J. Morgan, G. B. Ren, T. S. Cheng, S. E. Hooper, and C. T. Foxon, Semicond. Sci. Technol. 12, 55 (1997).

${ }^{13}$ D. J. Dewsnip, A. V. Andrianov, I. Harrison, J. W. Orton, D. E. Lacklison, G. B. Ren, S. E. Hooper, T. S. Cheng, and C. T. Foxon, Semicond. Sci. Technol. 13, 500 (1998).

${ }^{14}$ A. Salvador, W. Kim, Ö. Aktas, A. Botchkarev, Z. Fan, and H. Morkoç, Appl. Phys. Lett. 69, 2692 (1996).

${ }^{15}$ T. H. Myers et al. (unpublished).

${ }^{16}$ C. Ronning, E. P. Carlson, D. B. Thomson, and R. F. Davis, Appl. Phys. Lett. 73, 1622 (1998).

${ }^{17}$ C. Ronning, K. J. Linthicum, E. P. Carlson, P. J. Hartlieb, D. B. Thomson, T. Gehrke, and R. F. Davis, MRS Internet J. Nitride Semicond. Res. 4S1, G3.17 (1999).

${ }^{18}$ O. Brandt, H. Yang, H. Kostial, and K. H. Ploog, Appl. Phys. Lett. 69, 2707 (1996).

${ }^{19}$ K. H. Ploog and O. Brandt, J. Vac. Sci. Technol. A 16, 1609 (1998).

20 T. Yamamoto and H. Katayama-Yoshida, in Proceedings of the 19th International Conference on Defects in Semiconductors, Aveiro, Portugal, 1997, edited by G. Davies and M. H. Nazaré [Mater. Sci. Forum 258-263, 1185 (1997)].

${ }^{21}$ S. Nakamura, N. Iwasa, M. Senoh, and T. Mukai, Jpn. J. Appl. Phys., Part 1 31, 1258 (1992).

${ }^{22}$ J. Neugebauer and C. G. Van de Walle, Phys. Rev. Lett. 75, 4452 (1995).

${ }^{23}$ J. Neugebauer and C. G. Van de Walle, Appl. Phys. Lett. 68, 1829 (1996).

${ }^{24}$ P. Hohenberg and W. Kohn, Phys. Rev. 136, B864 (1964); W. Kohn and L. J. Sham, ibid. 140, A1133 (1965).

${ }^{25}$ M. Bockstedte, A. Kley, J. Neugebauer, and M. Scheffler, Comput. Phys. Commun. 107, 187 (1997).

26 J. Neugebauer and Chris G. Van de Walle, in Diamond, SiC and
Nitride Wide Bandgap Semiconductors, edited by C. H. Carter, Jr., G. Gildenblat, S. Nakamura, and R. J. Nemanich, Mater. Res. Soc. Symp. Proc. No. 339 (Materials Research Society, Pittsburgh, 1994), p. 687.

${ }^{27}$ S. G. Louie, S. Froyen, and M. L. Cohen, Phys. Rev. B 26, 1738 (1982).

${ }^{28}$ C. Stampfl and C. G. Van de Walle, Phys. Rev. B 59, 5521 (1999).

${ }^{29}$ H. J. Monkhorst and J. D. Pack, Phys. Rev. B 13, 5188 (1976).

${ }^{30}$ This correction was also taken into account in a previous publication (Ref. 5); however, there the correction was applied to the negative charge state, rather than the neutral charge state. This accounts for some of the differences between the results presented in Ref. 5 and in the present paper.

${ }^{31}$ D. B. Laks, C. G. Van de Walle, G. F. Neumark, P. E. Blöchl, and S. T. Pantelides, Phys. Rev. B 45, 10965 (1992).

${ }^{32}$ CRC Handbook of Chemistry and Physics, 73rd ed., edited by David R. Lide (CRC Press, Boca Raton, FL, 1992), pp. 5-18.

${ }^{33}$ J. E. Northrup (private communication).

${ }^{34}$ Lange's Handbook of Chemistry, 14th ed., edited by J. A. Dean (McGraw-Hill, New York, 1992), p. 6.72.

${ }^{35}$ Note that if we use the experimental value of $\Delta H_{f}\left[\mathrm{Be}_{3} \mathrm{~N}_{2}\right]$ to determine $\mu_{\mathrm{Be}}$, the calculated formation energy will be $0.2 \mathrm{eV}$ larger than if we use the calculated value of $\Delta H_{f}\left[\mathrm{Be}_{3} \mathrm{~N}_{2}\right]$. This small difference does not alter any of our conclusions, but may be kept in mind when comparing with the formation energies of other acceptors as calculated in Ref. 5, where experimental values for $\Delta H_{f}$ of the solubility-limiting compounds were used.

${ }^{36}$ Periodic Table of the Elements (Sargent-Welch Scientific Company, Skokie, 1980).

${ }^{37}$ Lange's Handbook of Chemistry (Ref. 34), p. 4.13.

${ }^{38}$ J. E. Northrup (unpublished).

${ }^{39}$ R. W. G. Wyckoff, Crystal Structures, 2nd ed. (Interscience Publishers, New York, 1964), Vol. 2, pp. 32-33.

${ }^{40}$ D. J. Chadi and K. J. Chang, Phys. Rev. Lett. 61, 873 (1988).

${ }^{41}$ J. Neugebauer and C. G. Van de Walle, Appl. Phys. Lett. 69, 503 (1996).

${ }^{42}$ C. G. Van de Walle, P. J. H. Denteneer, Y. Bar-Yam, and S. T. Pantelides, Phys. Rev. B 39, 10791 (1989).

${ }^{43}$ S. Limpijumnong and C. G. Van de Walle (unpublished).

${ }^{44}$ R. W. G. Wyckoff, Crystal Structures (Ref. 39), Vol. 1, pp. 108110.

${ }^{45}$ C. G. Van de Walle, Phys. Rev. B 57, R2033 (1998).

${ }^{46}$ E. J. Tarsa, B. Heying, X. H. Wu, P. Fini, S. P. DenBaars, and J. S. Speck, J. Appl. Phys. 82, 5472 (1997).

${ }^{47}$ M. Ilegems and R. Dingle, J. Appl. Phys. 44, 4234 (1973).

${ }^{48}$ F. Mireles and S. E. Ulloa, Phys. Rev. B 58, 3879 (1998). 